\title{
WEIGHTED FRACTIONAL BERNSTEIN'S INEQUALITIES AND THEIR APPLICATIONS
}

\author{
FENG DAI AND SERGEY TIKHONOV
}

\begin{abstract}
This paper studies the following weighted, fractional Bernstein inequality for spherical polynomials on $\mathbb{S}^{d-1}$ :

$$
\left\|\left(-\Delta_{0}\right)^{r / 2} f\right\|_{p, w} \leq C_{w} n^{r}\|f\|_{p, w}, \quad \forall f \in \Pi_{n}^{d},
$$

where $\Pi_{n}^{d}$ denotes the space of all spherical polynomials of degree at most $n$ on $\mathbb{S}^{d-1}$, and $\left(-\Delta_{0}\right)^{r / 2}$ is the fractional Laplacian-Beltrami operator on $\mathbb{S}^{d-1}$. A new class of doubling weights with conditions weaker than the $A_{p}$ is introduced, and used to fully characterize those doubling weights $w$ on $\mathbb{S}^{d-1}$ for which the weighted Bernstein inequality 0.1 holds for some $1 \leq p \leq \infty$ and all $r>\tau$. In the unweighted case, it is shown that if $0<p<\infty$ and $r>0$ is not an even integer, then (0.1) with $w \equiv 1$ holds if and only if $r>(d-1)\left(\frac{1}{p}-1\right)$. As applications, we show that any function $f \in L_{p}\left(\mathbb{S}^{d-1}\right)$ with $0<p<1$ can be approximated by the de la Vallée Poussin means of a Fourier-Laplace series, and establish a sharp Sobolev type Embedding theorem for the weighted Besov spaces with respect to general doubling weights.
\end{abstract}

\section{INTRODUCTION}

One of the fundamental results in analysis is the following Bernstein inequality for trigonometric polynomials:

$$
\left\|f^{(r)}\right\|_{p} \leq C n^{r}\|f\|_{p}, \quad 0<p \leq \infty, \quad r \in \mathbb{N}, \quad f \in \mathcal{T}_{n},
$$

where $\|\cdot\|_{p}=\|\cdot\|_{L^{p}[0,2 \pi]}, \mathcal{T}_{n}$ denotes the space of all trigonometric polynomials of degree at most $n$, and $C=1$ is known to be the best constant (see [1, p. 16, (4.4)]). In [22, p. 45, Theorem 4.1], Mastroianni and Totik established a weighted analogue of (1.1) for all doubling weights. Among other things, they proved that for any doubling weight $w$,

$$
\left\|f^{(r)}\right\|_{p, w} \leq C_{w} n^{r}\|f\|_{p, w}, \quad \forall f \in \mathcal{T}_{n}, \quad r \in \mathbb{N}, \quad 1 \leq p \leq \infty,
$$

where $\|f\|_{p, w}=\left\|f w^{1 / p}\right\|_{p}$, and $C_{w}$ depends only on the doubling constant of $w$. Later on, (1.2) was extended to the case of $0<p<1$ by Erdélyi [15, p. 69, Theorem 3.1].

For spherical polynomials on the unit sphere $\mathbb{S}^{d-1}$, it was shown in [7, Corollary 5.2 , p. 155] that if $r$ is an even integer and $w$ is a doubling weight, then the weighted

Date: October 17, 2018.

1991 Mathematics Subject Classification. 33C50, 33C52, 42B15, 42C10.

Key words and phrases. Weighted polynomial inequalities, polynomial approximation, sphere.

The first author was partially supported by the NSERC Canada under grant RGPIN 3116782010. The second author was partially supported by MTM 2011-27637, 2009 SGR 1303, RFFI 13-01-00043, and NSH-979.2012.1. 
Bernstein inequality,

$$
\left\|\left(-\Delta_{0}\right)^{r / 2} f\right\|_{p, w} \leq C_{w} n^{r}\|f\|_{p, w}, \quad \forall f \in \Pi_{n}^{d},
$$

holds for all $0<p \leq \infty$, where $\Pi_{n}^{d}$ denotes the space of all spherical polynomials of degree at most $n$ on $\mathbb{S}^{d-1}$, and $\Delta_{0}$ is the Laplacian-Beltrami operator on $\mathbb{S}^{d-1}$. In the unweighted case (i.e., $w=1$ ), (1.3) was shown earlier in 11, p.330, Theorem 3.2 ] for all $1 \leq p \leq \infty$.

The fractional Bernstein inequality, namely, the inequality (1.2) or (1.3) for positive $r$ that may not be an integer, plays an important role in harmonic analysis and PDE (see, for instance, [31, 32]), and the investigation of this inequality has a long history. Firstly, Lizorkin [21] showed that (1.1) holds for all $r>0$ and $1 \leq p \leq \infty$. ( A similar result for functions of exponential type was also established in [21]). Secondly, the fractional Bernstein inequality for trigonometric polynomials for $0<p<1$ was studied by Belinskii and Liflyand [3] who particularly observed that if $r>0$ is not an integer, then (1.1) does not hold for the full range of $0<p<1$. Of related interest is the fact that the (unweighted) fractional Bernstein inequality remains true in the $H^{p}$ spaces for all $0<p \leq 1$ and $r>0$. Finally, the fractional Bernstein inequality with $1 \leq p \leq \infty$ was established for multivariate trigonometric polynomials, and for spherical harmonics in [25, 26] and [11, 19, respectively.

In this paper, we shall study the weighted, fractional Bernstein inequality for spherical polynomials on $\mathbb{S}^{d-1}$ as well as its applications in approximation theory. We shall give a full characterization of all those doubling weights for which the weighted Bernstein inequality (1.3) holds for some $r \notin 2 \mathbb{N}$ and $1 \leq p \leq \infty$. It turns out that there is a considerable difference between the cases of integer power and non-integer power (i,e., fractional power) of the Laplace-Beltrami operator on the sphere. In fact, in the unweighted case, we prove the following.

Theorem 1.1. If $0<p<\infty, r>0$, and $d \geq 3$, then

$$
\begin{aligned}
& \sup _{f \in \Pi_{n}^{d},\|f\|_{p} \leq 1}\left\|\left(-\Delta_{0}\right)^{r / 2} f\right\|_{L^{p}\left(\mathbb{S}^{d-1}\right)} \\
& \sim \begin{cases}n^{r}, & \text { if } r>(d-1)\left(\frac{1}{p}-1\right) \text { or } r \in 2 \mathbb{N} ; \\
n^{r} \log ^{\frac{1}{p}} n, & \text { if } r=(d-1)\left(\frac{1}{p}-1\right), \text { and } r \notin 2 \mathbb{N} ; \\
n^{(d-1)\left(\frac{1}{p}-1\right)}, & \text { if } r<(d-1)\left(\frac{1}{p}-1\right), \text { and } r \notin 2 \mathbb{N} .\end{cases}
\end{aligned}
$$

According to Theorem 1.1 in the unweighted case (i.e., $w=1$ ), the Bernstein inequality (1.3) for a non-integer (i.e., fractional) power $r / 2$ of the Laplace-Beltrami operator holds if and only if $p>\frac{d-1}{d-1+r}$, whereas (1.3) for an integer power $r / 2$ holds for the full range of $0<p<\infty$.

We point out that in the case when $d=2$ and $r$ is not an integer, Theorem 1.1 is due to Belinskii and Liflyand [3], where the proofs do not seem to work for the higher-dimensional case.

The paper is organized as follows. Section 2 contains some preliminary results on spherical polynomial expansions on the unit sphere, as well as a technical theorem, Theorem [2.2. which gives sharp asymptotic estimates of the weighted norms of certain kernel functions. This theorem plays a crucial role in the proof of Theorem 1.1. whereas its proof is postponed to the appendix. Basic facts on doubling weights and several useful weighted polynomial inequalities are presented in Section 3. The fourth section is devoted to the proof of the fractional Bernstein inequality for spherical polynomials on $\mathbb{S}^{d-1}$. Theorem 1.1 as well as the weighted Bernstein 
inequality with doubling weights for $0<p \leq \infty$ are proved in this section. After that, in Section 5, we show that our method can yield a better result for weighted fractional Bernstein inequality with the Muckenhoupt $A_{p}$ weights.

One of our main results in this paper is given in Section 6 , where we prove a full characterization of the doubling weights for which the weighted Bernstein inequality holds. We introduce a new class $\mathbb{A}_{p, \tau}$ of weights on $\mathbb{S}^{d-1}$ and prove that the inequality (1.3) holds for any $r>\tau$ if and only if $w \in \mathbb{A}_{p, \tau}$. In particular, the inequality $\left\|f^{(r)}\right\|_{p, w} \leq C_{w} n^{r}\|f\|_{p, w}, 1 \leq p<\infty$, holds for a trigonometric polynomial $f \in \mathcal{T}_{n}$ for any $r>\tau$ if and only if $w \in \mathbb{A}_{p, \tau}$.

In Section 7, we consider spherical polynomial approximation in $L^{p}$ for $0<$ $p<1$, following the approach of Oswald for the trigonometric polynomials 23. In particular, we show that if $0<p<1$ and $f \in L^{p}\left(\mathbb{S}^{d-1}\right)$, then there exists a Fourier-Laplace series $\sigma$ on the sphere $\mathbb{S}^{d-1}$ such that the following quantitative estimate holds:

$$
\left\|f-V_{n} \sigma\right\|_{p} \leq C n^{-(d-1)\left(\frac{1}{p}-1\right)}\left(\sum_{k=1}^{n} k^{d-2-(d-1) p} E_{k}(f)_{p}^{p}\right)^{\frac{1}{p}},
$$

where $V_{n}$ is the de la Vallée Poussin operator, and $E_{k}(f)_{p}:=\inf _{g \in \Pi_{n}^{d}}\|f-g\|_{p}$. If, in addition, $\sum_{k=n+1}^{\infty} k^{(d-2)-(d-1) p} E_{k}(f)_{p}^{p}<\infty$, then $V_{n} f$ is well defined, and we have

$$
\left\|f-V_{n} f\right\|_{p} \leq C n^{-(d-1)\left(\frac{1}{p}-1\right)}\left(\sum_{k=n+1}^{\infty} k^{(d-2)-(d-1) p} E_{k}(f)_{p}^{p}\right)^{\frac{1}{p}} .
$$

In Section 8, we show how to apply our result to deduce the Sobolev-type embedding theorem for the weighted Besov spaces at the critical index. We prove that if $0<p<q \leq \infty, w$ is a doubling weight on $\mathbb{S}^{d-1}$, and $\alpha=s_{w}\left(\frac{1}{p}-\frac{1}{q}\right)$, then the weighted Besov space $B_{q}^{\alpha}\left(L_{p, w}\right)$ can be continuously embedded into the space $L_{q, w}$, where $s_{w}$ is a geometric constant depending only on $w$. (The precise definition of $s_{w}$ is given in Section 3). Examples will be given to show the index $\alpha=s_{w}\left(\frac{1}{p}-\frac{1}{q}\right)$, in general, is sharp. This result improves a result in [17, Cor. 4] and [10, Th. 2.5]. For the classical result, we refer to the paper of Peetre [24, (8.2)].

Finally, we prove the technical result, Theorem 2.2 , in appendix.

\section{Preliminaries}

Let $\mathbb{S}^{d-1}=\left\{x \in \mathbb{R}^{d}: \quad\|x\|=1\right\}$ denote the unit sphere of $\mathbb{R}^{d}$ endowed with the usual rotation-invariant measure $d \sigma(x)$, where, and in what follows, $\|x\|$ denotes the Euclidean norm of $x \in \mathbb{R}^{d}$. Let $\rho(x, y):=\arccos (x \cdot y)$ denote the usual geodesic distance of $x, y \in \mathbb{S}^{d-1}$, and $B(x, r):=\left\{y \in \mathbb{S}^{d-1}: \rho(x, y) \leq r\right\}$ the spherical cap centered at $x \in \mathbb{S}^{d-1}$ of radius $r \in(0, \pi]$. Given a constant $c>0$, we use the notation $c B:=B(x, c r)$ to denote the spherical cap with the same center as that of $B:=B(x, r)$ but $c$ times the radius of $B$. Given a set $E \subset \mathbb{S}^{d-1}$, we denote by $\chi_{E}$ and $|E|$ the characteristic function of $E$ and the Lebesgue measure $\sigma(E)$ of $E$, respectively. We shall use the notation $A \sim B$ to mean that there exists an inessential constant $c>0$, called the constant of equivalence, such that

$$
c^{-1} A \leq B \leq c A .
$$

For $0<p \leq \infty$ and $f \in L^{p}\left(\mathbb{S}^{d-1}\right)$, we define

$$
E_{n}(f)_{p}=\inf _{g \in \Pi_{n}}\|f-g\|_{p}, \quad n=0,1,2, \ldots
$$


A spherical polynomial of degree at most $n$ on $\mathbb{S}^{d-1}$ is the restriction to $\mathbb{S}^{d-1}$ of a polynomial in $d$ variables of total degree at most $n$. We denote by $\Pi_{n}^{d}$ the space of all real spherical polynomials of degree at most $n$ on $\mathbb{S}^{d-1}$. It is a finite dimensional vector space over $\mathbb{R}$ with $\operatorname{dim} \Pi_{n}^{d} \sim n^{d-1}$.

Let $\mathcal{H}_{0}^{d}$ denote the space of constant functions on $\mathbb{S}^{d-1}$. For each positive integer $n$, we denote by $\mathcal{H}_{n}^{d}$ the orthogonal complement of $\Pi_{n-1}^{d}$ in $\Pi_{n}^{d}$ with respect to the inner product of $L^{2}\left(\mathbb{S}^{d-1}\right)$. $\mathcal{H}_{n}^{d}$ is called the space of spherical harmonics of degree $n$ on $\mathbb{S}^{d-1}$. Thus, the spaces $\mathcal{H}_{n}^{d}, n=0,1, \cdots$ of spherical harmonics are mutually orthogonal with respect to the inner product of $L^{2}\left(\mathbb{S}^{d-1}\right)$, and for each $n \in \mathbb{N}$, $\operatorname{dim} \mathcal{H}_{n}^{d}=\operatorname{dim} \Pi_{n}^{d}-\operatorname{dim} \Pi_{n-1}^{d} \sim n^{d-2}$. Since the space of spherical polynomials is dense in $L^{2}\left(\mathbb{S}^{d-1}\right)$, each $f \in L^{2}\left(\mathbb{S}^{d-1}\right)$ has a spherical harmonic expansion:

$$
f=\sum_{k=0}^{\infty} \operatorname{proj}_{k} f
$$

where $\operatorname{proj}_{k}$ is the orthogonal projection of $L^{2}\left(\mathbb{S}^{d-1}\right)$ onto the space $\mathcal{H}_{k}^{d}$ of spherical harmonics, which has an integral representation:

$$
\operatorname{proj}_{k} f(x)=\frac{\Gamma\left(\frac{d-1}{2}\right)}{\Gamma(d-1)\left|\mathbb{S}^{d-1}\right|} \int_{\mathbb{S}^{d-1}} f(y) E_{k}^{\left(\frac{d-3}{2}, \frac{d-3}{2}\right)}(x \cdot y) d \sigma(y), \quad x \in \mathbb{S}^{d-1} .
$$

Here and elsewhere, we write

$$
\begin{aligned}
E_{k}^{(\alpha, \beta)}(t) & :=\frac{(2 k+\alpha+\beta+1) \Gamma(k+\alpha+\beta+1)}{\Gamma(k+\beta+1)} P_{k}^{(\alpha, \beta)}(t) \\
& =c_{\alpha, \beta} P_{n}^{(\alpha, \beta)}(1)\left\|P_{n}^{(\alpha, \beta)}\right\|_{2, \alpha, \beta}^{-2} P_{n}^{(\alpha, \beta)}(t),
\end{aligned}
$$

where $P_{k}^{(\alpha, \beta)}$ is the usual Jacobi polynomial of degree $k$ and indices $\alpha, \beta$, as defined in [28, Chapter IV], and

$$
\|g\|_{p, \alpha, \beta}:=\left(\int_{0}^{\pi}|g(\cos \theta)|^{p}(\sin \theta / 2)^{2 \alpha+1}(\cos \theta / 2)^{2 \beta+1} d t\right)^{\frac{1}{p}}, 0<p<\infty
$$

for $g:[-1,1] \rightarrow \mathbb{R}$. Furthermore, throughout the paper, we always assume that $\alpha \geq \beta \geq-\frac{1}{2}$.

Using (2.2), one can extend the definition of $\operatorname{proj}_{k}$ to the whole space $L^{1}\left(\mathbb{S}^{d-1}\right)$ so that there is a spherical harmonic expansion $f \backsim \sigma(f):=\sum_{k=0}^{\infty} \operatorname{proj}_{k}(f)$ associated to each $f \in L^{1}\left(\mathbb{S}^{d-1}\right)$. The series $\sigma(f)$ is called the Fourier-Laplace series of $f$ on $\mathbb{S}^{d-1}$. In the case of $d=2$, this is simply the usual Fourier series of $2 \pi$-periodic functions. If $d \geq 3$, then given any $1 \leq p \neq 2 \leq \infty$, there always exists a function $f \in L^{p}\left(\mathbb{S}^{d-1}\right)$ such that the partial sum of the Fourier-Laplace series $\sigma(f)$ does not converge in $L^{p}\left(\mathbb{S}^{d-1}\right)$ (see 4$]$ ). An important tool for the investigation of summability of the series $\sigma(f)$ is to use the Cesàro means of $\sigma(f)$, whose definition will be given below.

The Cesàro means of $\sigma(f)$ of order $\delta>-1$ are defined as usual by

$$
\sigma_{n}^{\delta}(f):=\sum_{k=0}^{n} \frac{A_{n-k}^{\delta}}{A_{n}^{\delta}} \operatorname{proj}_{k}(f), \quad n=0,1, \cdots,
$$

where $A_{k}^{\delta}=\frac{\Gamma(k+\delta+1)}{\Gamma(k+1) \Gamma(\delta+1)}$. It is known that if $\delta>\lambda:=\frac{d-2}{2}$, and $f \in L^{p}\left(\mathbb{S}^{d-1}\right)$ for $1 \leq p<\infty$ or $f \in C\left(\mathbb{S}^{d-1}\right)$ for $p=\infty$, then

$$
\lim _{n \rightarrow \infty}\left\|\sigma_{n}^{\delta} f-f\right\|_{p}=0 .
$$


This result, in particular, implies that if $f, g \in L^{1}\left(\mathbb{S}^{d-1}\right)$ satisfies $\operatorname{proj}_{j} f=\operatorname{proj}_{j} g$ for all $j \geq 0$ then one must have $f=g$.

Another approach to spherical harmonic analysis is through the Laplace-Beltrami operator $\Delta_{0}$ on $\mathbb{S}^{d-1}$ defined by

$$
\Delta_{0} f:=\left.\sum_{j=1}^{d} \frac{\partial^{2} F}{\partial x_{j}^{2}}\right|_{\mathbb{S}^{d-1}}, \quad \text { with } F(y):=f\left(\frac{y}{|y|}\right) .
$$

Indeed, each space $\mathcal{H}_{k}^{d}$ is the space of eigenfunctions of $\Delta_{0}$ corresponding to the eigenvalue $-\lambda_{k}=-k(k+d-2)$; namely,

$$
\mathcal{H}_{k}^{d}=\left\{f \in C^{2}\left(\mathbb{S}^{d-1}\right): \quad \Delta_{0} f=-\lambda_{k} f\right\}, \quad k=0,1, \cdots .
$$

Therefore, spherical harmonic polynomial expansions are simply the eigenvalue expansions of $\Delta_{0}$.

Given $r>0$, we define the fractional Laplace-Beltrami operator $\left(-\Delta_{0}\right)^{r}$ in a distributional sense by

$$
\operatorname{proj}_{k}\left[\left(-\Delta_{0}\right)^{r} f\right]=(k(k+d-2))^{r} \operatorname{proj}_{k}(f), \quad k=0,1, \cdots .
$$

Clearly, if $r=1$, this definition coincides with the definition given in (2.7).

Let $\eta$ be a nonnegative $C^{\infty}$-function on $\mathbb{R}$ with the properties that $\eta(x)=1$ for $|x| \leq 1$ and $\eta(x)=0$ for $|x| \geq 2$. For each integer $n \geq 1$, the generalized de la Vallée Poussin operator is defined by

$$
V_{n} f(x)=\sum_{k=0}^{2 n} \eta\left(\frac{k}{n}\right) \operatorname{proj}_{k} f(x)=\int_{\mathbb{S}^{d-1}} f(y) K_{n}(x \cdot y) d \sigma(y), \quad x \in \mathbb{S}^{d-1},
$$

where

$$
K_{n}(t)=C_{d} \sum_{k=0}^{2 n} \eta\left(\frac{k}{n}\right) E_{k}^{\left(\frac{d-3}{2}, \frac{d-3}{2}\right)}(t), \quad t \in[-1,1] .
$$

We will keep the notations $\eta, V_{n}$ and $K_{n}$ for the rest of the paper.

It turns out that the kernel $K_{n}$ in (2.11) is highly localized at the point $t=0$, as was shown in Lemma 2.1 below. To be more precise, we define, for a smooth cutoff function $\varphi:[0, \infty) \rightarrow \mathbb{C}$,

$$
B_{N, \varphi}^{(\alpha, \beta)}(t):=\sum_{k=0}^{\infty} \varphi\left(\frac{k}{N}\right) E_{k}^{(\alpha, \beta)}(t) .
$$

Then the following pointwise estimates of the kernels $B_{N, \varphi}^{(\alpha, \beta)}$ were known ( $[5$, Lemma 3.3] and [18, Theorem 2.6]):

Lemma 2.1. Let $\varphi \in C^{3 \ell-1}[0, \infty)$ be such that $\operatorname{supp} \varphi \subset[0,2]$ and $\varphi^{(j)}(0)=0$ for $j=1,2, \cdots, 3 \ell-2$. Then for the kernel function $B_{N} \equiv B_{N, \varphi}^{(\alpha, \beta)}$ defined by (2.12) with $\alpha \geq \beta \geq-1 / 2$,

$$
\left|B_{N}^{(i)}(\cos \theta)\right| \leq C_{\ell, i, \alpha}\left\|\varphi^{(3 \ell-1)}\right\|_{\infty} N^{2 \alpha+2 i+2}(1+N \theta)^{-\ell}, \quad i=0,1, \cdots,
$$

where $\theta \in[0, \pi], N \in \mathbb{N}, B_{N}^{(0)}(t)=B_{N, \varphi}^{(\alpha, \beta)}(t)$ and $B_{N}^{(i)}(t)=\left(\frac{d}{d t}\right)^{i}\left\{B_{N, \varphi}^{(\alpha, \beta)}(t)\right\}$ for $i \geq 1$. 
We conclude this section with a technical theorem, which gives a sharp asymptotic estimate of the weighted $L^{p}$ norm of the following kernel function:

$$
G_{n, r}^{(\alpha, \beta)}(t):=\sum_{k=0}^{\infty} \eta\left(\frac{k}{n}\right)(k(k+\alpha+\beta+1))^{\frac{r}{2}} E_{k}^{(\alpha, \beta)}(t), \quad r \geq 0 .
$$

For simplicity, we will write $G_{n, r}$ for $G_{n, r}^{(\alpha, \beta)}$, and $G_{n}$ for $G_{n, 0}$, whenever $\alpha, \beta$ are understood and no confusion is possible from the context. Recall that the norm $\|g\|_{p, \alpha, \beta}$ is defined by (2.4).

Theorem 2.2. Let $G_{n, r} \equiv G_{n, r}^{(\alpha, \beta)}$ be defined by (2.14), and let $0<p<1$ and $r>0$. Assume that $r$ is not an even integer if $\alpha+\beta+1>0$, and $r$ is not an integer if $\alpha+\beta+1=0$. Then

$$
\frac{\left\|G_{n, r}\right\|_{p, \alpha, \beta}}{\left\|G_{n}\right\|_{p, \alpha, \beta}} \sim \begin{cases}n^{r}, & \text { if } r>(2 \alpha+2)\left(\frac{1}{p}-1\right), \\ n^{(2 \alpha+2)\left(\frac{1}{p}-1\right)}, & \text { if } r<(2 \alpha+2)\left(\frac{1}{p}-1\right), \\ n^{(2 \alpha+2)\left(\frac{1}{p}-1\right)} \log ^{\frac{1}{p}} n, & \text { if } r=(2 \alpha+2)\left(\frac{1}{p}-1\right) .\end{cases}
$$

Theorem 2.2 will play a crucial role in the proof of Theorem 1.1, whereas its proof is quite technical. To avoid interruption of our later discussion of various polynomial inequalities, we postpone the proof of this theorem to the appendix section.

More results on spherical harmonic expansions can be found in the book 30.

\section{Weighted POLYNOMial INEQUALITIES}

In this section, we will review some known facts and results concerning doubling weights, which will be useful in the remaining sections of the paper.

3.1. Doubling weights and properties. Given a weight function $w$ on $\mathbb{S}^{d-1}$, we write $w(E):=\int_{E} w(x) d \sigma(x)$ for a measurable $E \subset \mathbb{S}^{d-1}$, and denote by $L_{p, w} \equiv$ $L_{p, w}\left(\mathbb{S}^{d-1}\right)$ the space of all real functions $f$ on $\mathbb{S}^{d-1}$ with finite quasi- norm

$$
\|f\|_{p, w}:= \begin{cases}\left(\int_{\mathbb{S}^{d-1}}|f(x)|^{p} w(x) d \sigma(x)\right)^{\frac{1}{p}}, & 0<p<\infty, \\ \underset{x \in \mathbb{S}^{d-1}}{\operatorname{esssup}}|f(x)|, & p=\infty .\end{cases}
$$

A weight function $w$ on $\mathbb{S}^{d-1}$ is said to satisfy the doubling condition if there exists a constant $L>0$ such that

$$
w(2 B) \leq L w(B) \text { for all spherical caps } B \subset \mathbb{S}^{d-1},
$$

where the least constant $L$ is called the doubling constant of $w$, and is denoted by $L_{w}$. Following [22, we set, for a given doubling weight $w$ on $\mathbb{S}^{d-1}$,

$$
w_{n}(x)=n^{d-1} \int_{B\left(x, \frac{1}{n}\right)} w(y) d \sigma(y), \quad n=1,2, \ldots, \quad \text { and } w_{0}(x)=w_{1}(x) .
$$

Define

$$
s_{w}^{\prime}:=\inf \left\{s \geq 0: \sup _{m \in \mathbb{N}} \sup _{B} \frac{w\left(2^{m} B\right)}{2^{m s} w(B)}<\infty\right\}
$$


where the second supremum on the right is taken over all spherical caps $B \subset \mathbb{S}^{d-1}$. It can be shown that the number $s_{w}^{\prime}$ exits and satisfies

$$
\varlimsup_{m \rightarrow \infty} \frac{1}{m} \log _{2}\left(\sup _{B} \frac{w\left(2^{m} B\right)}{w(B)}\right) \leq s_{w}^{\prime} \leq \frac{\log L_{w}}{\log 2} .
$$

We remark that in many cases the infimum in (3.3) is attained at $s_{w}^{\prime}$ and is computable. Taking the simple case

$$
w(x)=\left|x_{1}\right|^{\alpha_{1}} \cdots\left|x_{d}\right|^{\alpha_{d}}, \min _{1 \leq j \leq d} \alpha_{j} \geq 0
$$

for example, one has (see, e.g., [10, (1.9)])

$$
s_{w}^{\prime}:=d-1+\sum_{j=1}^{d} \alpha_{j}-\min _{1 \leq j \leq d} \alpha_{j} .
$$

From now on, we always assume that $w$ is a doubling weight on $\mathbb{S}^{d-1}$ normalized by $\int_{\mathbb{S}^{d-1}} w(y) d \sigma(y)=1$, we set $s_{w}=s_{w}^{\prime}$ if the infimum in (3.3) is achieved at $s_{w}^{\prime}$, and otherwise, we set $s_{w}$ to be a fixed constant satisfying $s_{w}^{\prime}<s_{w} \leq \log L_{w} / \log 2$. Unless otherwise stated, all general constants $C$ below depend only on $L_{w}$, and the expression

$$
\sup _{m \in \mathbb{N}} \sup _{B} \frac{w\left(2^{m} B\right)}{2^{m s_{w}} w(B)}
$$

whenever a doubling weight is involved.

Using (3.2) and (3.3), one can easily seen that

$$
w_{n}(x) \leq C 2^{s_{w}}(1+n \rho(x, y))^{s_{w}} w_{n}(y), \quad x, y \in \mathbb{S}^{d-1}, \quad n \geq 0 .
$$

The following lemma collects some useful properties on doubling weights:

Lemma 3.1. 7, Section 2] Let $w$ be a doubling weight on $\mathbb{S}^{d-1}$.

(i) If $0<r<t$ and $x \in \mathbb{S}^{d-1}$, then

$$
w(B(x, t)) \leq C\left(\frac{t}{r}\right)^{s_{w}} w(B(x, r))
$$

(ii) For $x, y \in \mathbb{S}^{d-1}$ and $n=0,1, \cdots$,

$$
w_{n}(x) \leq C(1+n \rho(x, y))^{s_{w}} w_{n}(y) .
$$

The following theorem was proved in [7, Corollary 3.4].

Theorem 3.2. For $f \in \Pi_{n}^{d}$ and $0<p<\infty$,

$$
C^{-1}\|f\|_{p, w_{n}} \leq\|f\|_{p, w} \leq C\|f\|_{p, w_{n}},
$$

where $C>0$ depends only on $d, L_{w}$ and $p$ when $p$ is small.

\subsection{A maximal function for spherical polynomials.}

Definition 3.3. [7, (3.1)] Given $\xi>0, f \in C\left(\mathbb{S}^{d-1}\right)$ and $n \in \mathbb{Z}_{+}$, we define

$$
f_{\xi, n}^{*}(x)=\max _{y \in \mathbb{S}^{d-1}}|f(y)|(1+n \rho(x, y))^{-\xi}, \quad x \in \mathbb{S}^{d-1} .
$$

Theorem 3.4. [7], Theorem 3.1] If $0<p \leq \infty, f \in \Pi_{n}^{d}$ and $\xi>\frac{s_{w}}{p}$, then

$$
\|f\|_{p, w} \leq\left\|f_{\xi, n}^{*}\right\|_{p, w} \leq C\|f\|_{p, w}
$$

where $C>0$ depends only on $d, L_{w}$ and $\xi$. 
3.3. Weighted cubature formulas and polynomial inequalities. We start with the following definition.

Definition 3.5. A subset $\Lambda$ of $\mathbb{S}^{d-1}$ is called $\varepsilon$-separated for some $\varepsilon>0$ if $\rho\left(\omega, \omega^{\prime}\right) \geq \varepsilon$ for any two distinct points $\omega, \omega^{\prime} \in \Lambda$. A $\varepsilon$-separated subset $\Lambda$ of $\mathbb{S}^{d-1}$ is called maximal if $\mathbb{S}^{d-1}=\bigcup_{\omega \in \Lambda} B(\omega, \varepsilon)$.

From now on, let $\delta_{0}$ be a sufficiently small constant depending only on $L_{w}$.

Lemma 3.6. 77, Theorems 4.1][10, Lemma 3.4] Given any maximal $\frac{\delta}{n}$-separated subset $\Lambda$ of $\mathbb{S}^{d-1}$ with $\delta \in\left(0, \delta_{0}\right]$, there exist positive numbers $\lambda_{\omega} \sim w\left(B\left(\omega, \frac{1}{N}\right)\right)$, $\omega \in \Lambda$, such that the following are true:

$$
\int_{\mathbb{S}^{d-1}} f(x) w(x) d \sigma(x)=\sum_{\omega \in \Lambda} \lambda_{\omega} f(\omega), \quad \forall f \in \Pi_{4 n}^{d},
$$

and

$$
\|f\|_{p, w} \sim \begin{cases}\left(\sum_{\omega \in \Lambda} \lambda_{\omega}|f(\omega)|^{p}\right)^{\frac{1}{p}}, & 0<p<\infty, \\ \max _{\omega \in \Lambda}|f(\omega)|, & p=\infty,\end{cases}
$$

where the constants of equivalence depend only on $L_{w}$, and $p$ when $p$ is small.

Lemma 3.7. 10, Lemma 2.3] If $0<p<q \leq \infty$, then

$$
\|f\|_{q, w} \leq C n^{\left(\frac{1}{p}-\frac{1}{q}\right) s_{w}}\|f\|_{p, w}, \quad \forall f \in \Pi_{n}^{d} .
$$

\section{The Bernstein inequality with Doubling Weights}

In this section we study the sharp Bernstein inequality, that is, a sharp growth on $n$ of the following expression:

$$
\sup _{f \in \Pi_{n}^{d},\|f\|_{p} \leq 1}\left\|\left(-\Delta_{0}\right)^{r / 2} f\right\|_{L^{p}\left(\mathbb{S}^{d-1}\right)}
$$

or, more generally,

$$
\sup _{f \in \Pi_{n}^{d},\|f\|_{p, w} \leq 1}\left\|\left(-\Delta_{0}\right)^{r / 2} f\right\|_{p, w} .
$$

Theorem 1.1 in the introduction gives an answer to the first question, that is, in the unweighted case. In the case of $d=2$, this result (for $0<p<1$ ) is due to Belinskii and Liflyand [3], but their proof, especially for the lower estimates, does not work for the case of higher-dimensional spheres.

For the proof of Theorem 1.1 we first note that the lower estimates in (1.4) of Theorem 1.1 follow directly from Theorem 2.2 with $\alpha=\beta=\frac{d-3}{2}$. For the upper estimates in (1.4), we shall prove a more general weighted result for all doubling weights.

Theorem 4.1. If $d \geq 3,0<p<\infty, r>0$, and $w$ is a doubling weight on $\mathbb{S}^{d-1}$, then

$$
\sup _{f \in \Pi_{n}^{d},\|f\|_{p, w} \leq 1}\left\|\left(-\Delta_{0}\right)^{r / 2} f\right\|_{p, w} \leq C \Phi(n, r, p)
$$


where

$$
\Phi(n, r, p)= \begin{cases}n^{r}, & \text { if } r>\delta(p, w) \text { or } r \in 2 \mathbb{N} \\ n^{r}(\log n)^{\max \left\{\frac{1}{p}, 1\right\}}, & \text { if } r=\delta(p, w) ; \\ n^{\delta(p, w)}, & \text { if } r<\delta(p, w),\end{cases}
$$

and

$$
\delta(p, w):= \begin{cases}\frac{s_{w}}{p}-(d-1), & \text { if } 0<p \leq 1 ; \\ \frac{s_{w}-(d-1)}{p}, & \text { if } 1<p<\infty .\end{cases}
$$

Remark 4.2. (i) The proof of Theorem 4.1 below works equally well when $d=2$ and $r$ is not an integer, in which case (4.1) is simply the usual Bernstein inequality for the fractional derivatives of trigonometric polynomials, and to the best of our knowledge, our results for general doubling weights and non-integer $r$ are new. Note also that in the case of $w=1$ (i.e., the unweighted case), $s_{w}=d-1$. Thus, the upper estimate of (1.4) is a direct consequence of Theorem 4.1.

(ii) Note that in the case when the power $r / 2$ of the Laplace-Beltrami operator is an integer, then the weighted Bernstein inequality (1.3) holds for the full range of $0<p<\infty$, whereas in the case of non-integer power, this is no longer true.

The proof of Theorem 4.1 given below is different from that of [3].

Proof of Theorem 4.1. Assume that $2^{m-1} \leq n<2^{m}$. Define $L_{0} g=V_{1} g$, and $L_{j} g=V_{2^{j}} f-V_{2^{j-1}} f$ for $j \geq 1$. Then for any $f \in \Pi_{n}^{d}$,

$$
\left(-\Delta_{0}\right)^{r / 2} f=\left(-\Delta_{0}\right)^{r / 2}\left(V_{2^{m}} f\right)=\sum_{j=0}^{m}\left(-\Delta_{0}\right)^{r / 2} L_{j} f .
$$

However, using (2.2) and (2.9), it is easily seen that

$$
\left(-\Delta_{0}\right)^{r / 2} L_{j} f(x)=\int_{\mathbb{S}^{d-1}} f(y) L_{j, r}(x \cdot y) d \sigma(y),
$$

where $L_{0, r}(t)=c_{d, r} E_{1}^{\left(\frac{d-3}{2}, \frac{d-3}{2}\right)}(t)$,

$$
L_{j, r}(t)=c \sum_{k=2^{j}}^{2^{j+2}} \psi\left(2^{-j} k\right)(k(k+d-2))^{r / 2} E_{k}^{\left(\frac{d-3}{2}, \frac{d-3}{2}\right)}(t), \quad j \geq 1,
$$

and $\psi(x)=\eta(x / 2)-\eta(x)$. Invoking Lemma 2.1 with $\varphi(x)=\psi(x)\left(x\left(x+2^{-j}(d-\right.\right.$ 2)) $)^{r / 2}$, we have

$$
\left|L_{j, r}(\cos \theta)\right| \leq c 2^{j r+d-1}\left(1+2^{j} \rho(x, y)\right)^{-\ell}, \quad \forall \ell>1 .
$$

Recalling the definition of $w_{k}(x)$ in (3.2), we obtain that for $0<p \leq 1$,

$$
\begin{aligned}
& \left|\left(-\Delta_{0}\right)^{r / 2} L_{j} f(x)\right|^{p} w_{2^{j}}(x) \\
& \leq c n^{(d-1)(1-p)} \int_{\mathbb{S}^{d-1}}|f(y)|^{p}\left|L_{j, r}(x \cdot y)\right|^{p} d \sigma(y) w_{2^{j}}(x) \\
& \leq c n^{(d-1)(1-p)} 2^{j p(d-1+r)} \int_{\mathbb{S}^{d-1}}|f(y)|^{p}\left(1+2^{j} \rho(x, y)\right)^{-p \ell+s_{w}} w_{2^{j}}(y) d \sigma(y),
\end{aligned}
$$

where we used (4.3) and the unweighted Nikolskii inequality (i.e., Lemma 3.7 with $\left.s_{w}=d-1\right)$ in the first step, and used (4.4) and (3.6) in the second step. Integrating 
this last inequality with respect to $x \in \mathbb{S}^{d-1}$ gives

$$
\begin{aligned}
& \left\|\left(-\Delta_{0}\right)^{r / 2} L_{j} f\right\|_{p, w}^{p} \sim\left\|\left(-\Delta_{0}\right)^{r / 2} L_{j} f\right\|_{p, w_{2^{j}}}^{p} \\
& \leq C n^{-(d-1) p} 2^{j p(d-1+r)}\left(n 2^{-j}\right)^{d-1} \int_{\mathbb{S}^{d-1}}|f(y)|^{p} w_{2^{j}}(y) d \sigma(y) \\
& \leq C n^{-(d-1) p} 2^{j p(d-1+r)}\left(n 2^{-j}\right)^{s_{w}} \int_{\mathbb{S}^{d-1}}|f(y)|^{p} w_{n}(y) d \sigma(y) \\
& \leq C n^{s_{w}-p(d-1)} 2^{j\left(p(d-1+r)-s_{w}\right)}\|f\|_{p, w}^{p},
\end{aligned}
$$

where the first step uses Theorem 3.2 and the fact that $\left(-\Delta_{0}\right)^{r / 2} L_{j} f \in \Pi_{2^{j}}^{d}$. The second step uses the inequality (4.5) with $\ell>\left(s_{w}+d-1\right) / p$, the third step uses (3.5), and the last step follows from Theorem 3.2 and the fact that $f \in \Pi_{n}^{d}$. Thus, combining (4.2) with (4.6), we obtain

$$
\left\|\left(-\Delta_{0}\right)^{r / 2}\left(L_{j} f\right)\right\|_{p}^{p} \leq C\left[n^{s_{w}-p(d-1)} \sum_{j=0}^{m} 2^{j\left(p(d-1+r)-s_{w}\right)}\right]\|f\|_{p, w}^{p},
$$

which, by straightforward calculation gives the desired upper bound.

The case of $p>1$ can be treated similarly. Indeed, instead of using Nikolskii's inequality, we use Hölder's inequality to obtain

$$
\begin{aligned}
& \left|\left(-\Delta_{0}\right)^{r / 2} L_{j} f(x)\right|^{p} w_{2^{j}}(x) \leq C 2^{j r(p-1)} \int_{\mathbb{S}^{d-1}}|f(y)|^{p} \mid L_{j, r}(x \cdot y) d \sigma(y) w_{2^{j}}(x) \\
& \leq C 2^{j r p} 2^{j(d-1)} \int_{\mathbb{S}^{d-1}}|f(y)|^{p}\left(1+2^{j} \rho(x, y)\right)^{-\ell+s_{w}} w_{2^{j}}(y) d \sigma(y) \\
& \leq C 2^{j r p}\left(\frac{2^{j}}{n}\right)^{d-1-s_{w}} 2^{j(d-1)} \int_{\mathbb{S}^{d-1}}|f(y)|^{p}\left(1+2^{j} \rho(x, y)\right)^{-\ell+s_{w}} w_{n}(y) d \sigma(y) .
\end{aligned}
$$

We then integrate the last inequality with respect to $x \in \mathbb{S}^{d-1}$ and deduce

$$
\left\|\left(-\Delta_{0}\right)^{r / 2} L_{j} f\right\|_{p, w} \leq C 2^{j r}\left(\frac{2^{j}}{n}\right)^{\left(d-1-s_{w}\right) / p}\|f\|_{p, w},
$$

which, in turn, implies

$$
\left\|\left(-\Delta_{0}\right)^{r / 2}\left(L_{j} f\right)\right\|_{p} \leq \sum_{j=0}^{m}\left\|\left(-\Delta_{0}\right)^{r / 2} L_{j} f\right\|_{p, w} \leq C\left(\sum_{j=0}^{m} 2^{j r}\left(\frac{2^{j}}{n}\right)^{\left(d-1-s_{w}\right) / p}\right)\|f\|_{p, w} .
$$

The desired upper bounds for the case of $p>1$ then follow.

\section{The Bernstein inequality with $A_{p}$ Weights}

Given $1<p<\infty$, we say a weight function $w$ on $\mathbb{S}^{d-1}$ belongs to $A_{p}$ if

$$
\sup _{B} \frac{w(B)}{|B|}\left(\frac{1}{|B|} \int_{B} w(x)^{-\frac{p^{\prime}}{p}} d \sigma(x)\right)^{p-1} \leq A_{p}(w)<\infty
$$

where the supremum is taken over all the spherical caps $B$ of $\mathbb{S}^{d-1}$. A characterization of the Muckenhoupt $A_{p}$ condition was recently obtained in [20, Th. 2.4].

Similarly, a weight function $w$ belongs to $A_{1}$ if there exists a constant $A_{1}(w)>0$ such that for all spherical caps $B \subset \mathbb{S}^{d-1}$,

$$
\frac{1}{|B|} \int_{B} w(x) d \sigma(x) \leq A_{1}(w) \inf _{x \in B} w(x) .
$$


It is well known that if $1<p<\infty$ and $w \in A_{p}$ then

$$
\|M f\|_{p, w} \leq C_{p}\|f\|_{p, w},
$$

where $M f$ denotes the Hardy-Littlewood maximal function on $\mathbb{S}^{d-1}$ :

$$
M f(x):=\sup _{0<r<\pi} \frac{1}{|B(x, r)|} \int_{B(x, r)}|f(y)| d \sigma(y) .
$$

Another useful fact on $A_{p}$ weights is the following: if $w \in A_{p}$ and $1 \leq p<\infty$, then one can choose $s_{w}$ so that

$$
s_{w} \leq p(d-1)
$$

see [27, p. 196, (5)]. Let us also mention that the $A_{p}$ classes have a self-improvement property ([27, p. 202]), that is, if $w \in A_{p}$ for some $1<p<\infty$, then $w \in A_{p-\epsilon}$ for some $\epsilon>0$.

Using properties of the $A_{p}$-weights and Theorem 4.1, we can easily deduce the following weighted Bernstein inequality for $A_{p}$ weights:

Theorem 5.1. If $1 \leq p<\infty, r>0, q:=\max \left\{p, \frac{d-1+p r}{d-1}\right\}$, and $w \in A_{q}$, then

$$
\left\|\left(-\Delta_{0}\right)^{r / 2} f\right\|_{p, w} \leq C_{A_{q}(w)} n^{r}\|f\|_{p, w}, \quad \forall f \in \Pi_{n}^{d}
$$

This, in particular, implies that if $w \in A_{p}$, then (5.5) holds for all $r>0$.

Proof. Firstly, we show (5.5) for the case of $r>\left(1-\frac{1}{p}\right)(d-1)$. In this case, $q=\frac{d-1+p r}{d-1}$. Since $w \in A_{q}$ implies that $w \in A_{q-\varepsilon}$ for some small $\varepsilon>0$, using (5.4), we deduce that $s_{w}<q(d-1)=d-1+p r$, or equivalently, $r>\frac{s_{w}-(d-1)}{p}$. The desired inequality (5.5) in this case then follows from Theorem 4.1.

Next, we show (5.5) for $0<r \leq\left(1-\frac{1}{p}\right)(d-1)$, in which case $q=p$. If $p=1$ and $w \in A_{1}$ then using (5.4), we have $s_{w}=d-1$, and according to Theorem 4.1. (5.5) holds whenever $r>s_{w}-(d-1)=0$. Thus, it remains to show (5.5) for the case of $w \in A_{p}$ and $1<p<\infty$. Observe that for all $f \in \Pi_{n}^{d}$,

$$
\left(-\Delta_{0}\right)^{r / 2} f(x)=\left(-\Delta_{0}\right)^{r / 2} V_{n} f(x):=\int_{\mathbb{S}^{d-1}} f(y) K_{n, r}(x \cdot y) d \sigma(y),
$$

where

$$
K_{n, r}(\cos \theta):=C_{d} \sum_{k=0}^{2 n} \eta\left(\frac{k}{n}\right) E_{k}^{\left(\frac{d-3}{2}, \frac{d-3}{2}\right)}(\cos \theta) .
$$

Using Lemma 9.2 with $\alpha=\beta=\frac{d-3}{2}$, we have

$$
\left|K_{n, r}(\cos \theta)\right| \leq c n^{d-1+r}(1+n \theta)^{-(d-1+r)}, \quad 0 \leq \theta \leq \pi .
$$

Thus, a straightforward computation, using (5.6), shows that for all $f \in \Pi_{n}^{d}$,

$$
\left|\left(-\Delta_{0}\right)^{r / 2} f(x)\right| \leq c n^{r} M f(x), \quad x \in \mathbb{S}^{d-1} .
$$

Since $w \in A_{p}$ and $1<p<\infty$, this implies that

$$
\left\|\left(-\Delta_{0}\right)^{r / 2} f\right\|_{p, w} \leq c n^{r}\|M f\|_{p, w} \leq c n^{r}\|f\|_{p, w},
$$

which is the desired Bernstein inequality. 


\section{Weighted Characterization of the Bernstein inequality}

Definition 6.1. Given $1<p<\infty$, and $\tau \geq 0$, we say a weight function $w$ on $\mathbb{S}^{d-1}$ belongs to the class $\mathbb{A}_{p, \tau}$ if for any $r>\tau$,

$$
\sup _{B} \sup _{n \in \mathbb{N}} \frac{w_{n}(B)}{|B|}\left(\frac{1}{|B|} \int_{B} w_{n}(y)^{-\frac{1}{p-1}} d \sigma(y)\right)^{p-1}\left(1+n|B|^{\frac{1}{d-1}}\right)^{-r p}=\mathbb{A}_{p, \tau}(w)<\infty,
$$

where the first supremum is taken over all spherical caps of $\mathbb{S}^{d-1}$. We say $w \in \mathbb{A}_{1, \tau}$ if there exists a constant $C>0$ such that for all spherical caps $B \subset \mathbb{S}^{d-1}$, and all $r>\tau$,

$$
\frac{w_{n}(B)}{|B|} \leq C\left(1+n|B|^{\frac{1}{d-1}}\right)^{r} \inf _{x \in B} w_{n}(x) .
$$

The smallest value of $C$ in (6.2) is called the $\mathbb{A}_{1, \tau}(w)$ constant.

The following lemma collects some useful properties on weights from the class $\mathbb{A}_{p, \tau}$.

Lemma 6.2. (i) If $0<\tau_{1} \leq \tau_{2}$ and $1 \leq p<\infty$, then $\mathbb{A}_{p, \tau_{1}} \subset \mathbb{A}_{p, \tau_{2}}$.

(ii) If $1 \leq p \leq q<\infty$ and $\tau>0$, then $\mathbb{A}_{p, \tau} \subset \mathbb{A}_{q, \tau}$.

(iii) If $w$ is a doubling weight on $\mathbb{S}^{d-1}$, then $w \in \mathbb{A}_{p, \tau}$ with $\tau:=\frac{s_{w}-(d-1)}{p}$.

(iv) For any $1 \leq p<\infty$, we have

$$
A_{p} \subset \bigcup_{\tau>0} \mathbb{A}_{p, \tau} .
$$

(v) $w \in \mathbb{A}_{p, \tau}$ if and only if for any $f \in L\left(\mathbb{S}^{d-1}\right)$, any spherical cap $B:=B(x, \theta) \subset$ $\mathbb{S}^{d-1}$, and any $r>\tau$,

$$
\left|f_{B}\right|^{p} \leq C(1+n \theta)^{r p} \frac{1}{w_{n}(B)} \int_{B}|f(y)|^{p} w_{n}(y) d \sigma(y),
$$

where $f_{B}:=\frac{1}{|B|} \int_{B} f(y) d \sigma(y)$, and the constant $C$ is independent of $B, f$ and $n$.

Proof. Assertion (i) is obvious from the definition of the $\mathbb{A}_{p, \tau}$ class. Assertion (ii) follows by Hölder's inequality and the fact that the term on the left hand side of (6.1) is a decreasing function of $p$.

To prove Assertion (iii) for the case of $p>1$, it suffices to show that for $B=$ $B(x, \theta) \subset \mathbb{S}^{d-1}$, and $\tau:=\frac{s_{w}-(d-1)}{p}$,

$$
\frac{w_{n}(B)}{|B|}\left(\frac{1}{|B|} \int_{B} w_{n}(y)^{-\frac{1}{p-1}} d \sigma(y)\right)^{p-1} \leq C(1+n \theta)^{\tau p} .
$$

(6.4) holds trivially if $\theta \leq \frac{1}{n}$ since $w_{n}(y) \sim w_{n}(z)$ whenever $\rho(y, z) \leq n^{-1}$. Now assume that $\frac{1}{\theta} \sim m$ for some positive integer $m \leq n$. Then $w_{m}(y) \sim w_{m}(x)$ whenever $y \in B$. Since $m \leq n$, it is easily seen that

$$
w_{n}(B) \sim w(B) \sim|B| w_{m}(x),
$$

and using Lemma 3.1 we deduce

$$
\frac{w_{n}(y)}{w_{m}(y)} \geq c\left(\frac{n}{m}\right)^{d-1-s_{w}}, \quad y \in \mathbb{S}^{d-1} .
$$


Thus,

$$
\begin{aligned}
& \frac{w_{n}(B)}{|B|}\left(\frac{1}{|B|} \int_{B} w_{n}(y)^{-\frac{1}{p-1}} d \sigma(y)\right)^{p-1} \\
& \quad \leq c w_{m}(x)\left(\frac{1}{|B|} \int_{B} w_{m}(y)^{-\frac{1}{p-1}} d \sigma(y)\right)^{p-1}\left(\frac{n}{m}\right)^{s_{w}-(d-1)} \\
& \quad \leq c\left(\frac{n}{m}\right)^{s_{w}-(d-1)} \sim(n \theta)^{s_{w}-d+1} \leq c(n \theta)^{r p},
\end{aligned}
$$

provided that $r \geq\left(s_{w}-d+1\right) / p$. This proves Assertion (iii) for the case $p>1$. Assertion (iii) for the case $p=1$ can be treated similarly.

Assertion (iv) follows directly from Theorem 5.1 and Theorem 6.4 below.

Finally, we show assertion (v). We first prove the necessity. Again we just deal with the case of $p>1$ for the sake of simplicity. Using Hölder's inequality and the $\mathbb{A}_{p, \tau}$-condition, we have, for $r>\tau$,

$$
\begin{aligned}
\left|f_{B}\right|^{p} & \leq\left(\frac{1}{|B|} \int_{B}|f(y)| w_{n}(y)^{\frac{1}{p}} w_{n}(y)^{-\frac{1}{p}} d \sigma(y)\right)^{p} \\
& \leq\left(\frac{1}{|B|} \int_{B}|f(y)|^{p} w_{n}(y) d \sigma(y)\right)\left(\frac{1}{|B|} \int_{B} w_{n}(y)^{-\frac{p^{\prime}}{p}} d \sigma(y)\right)^{p-1} \\
& \leq c\left(1+n|B|^{\frac{1}{d-1}}\right)^{r p} \frac{1}{w_{n}(B)} \int_{B}|f(y)|^{p} w_{n}(y) d \sigma(y) .
\end{aligned}
$$

This proves that the $\mathbb{A}_{p, \tau}$-condition (6.1) implies the condition (6.3). Finally, the sufficiency part of Assertion (v) follows directly by setting $f(x)=w_{n}(x)^{-\frac{1}{p-1}}$.

The next result was proved in [8, Lemma 2.5].

Lemma 6.3. If $1 \leq p<\infty$, and $w$ is a doubling weight, then

$$
\left\|V_{n} f\right\|_{p, w_{n}} \leq c\|f\|_{p, w_{n}}, \quad \forall f \in L_{p}, \quad \forall n \in \mathbb{N} .
$$

Before stating the main result in this section, we recall that, if the power $r / 2$ is a positive integer, then for all doubling weights $w$, the weighted Bernstein inequality (1.3) holds for the full range of $0<p<\infty$, while this is no longer true when the power $r / 2$ is non-integer. Indeed, for the latter case, we have the following main theorem, which characterizes those weights $w$ for which the weighted Bernstein inequality (1.3) holds.

Theorem 6.4. Assume that $1 \leq p<\infty$, w is a doubling weight on $\mathbb{S}^{d-1}$, and $\tau \geq 0$. Then the weighted Bernstein inequality (1.3), with the constant $C$ independent of $n$ and $f$, holds for all $r>\tau$ if and only if $w \in \mathbb{A}_{p, \tau}$.

Remark 6.5. Note that Theorem 6.4 is new even in the case of trigonometric polynomials (i.e., $d=2$ ). Next, we would like to remark that the sufficiency part of this theorem implies Theorem 4.1 for $1 \leq p<\infty$. Indeed, if $w$ is a doubling weight, then by Lemma 6.2 (iii), $w \in \mathbb{A}_{p, \tau}$ with $\tau:=\frac{s_{w}-(d-1)}{p}$ and by Theorem 6.4, the Bernstein inequality (5.5) holds.

Proof. Firstly, we show that if the weighted Bernstein inequality (5.5) holds for some positive $r \notin 2 \mathbb{N}$, then $w \in \mathbb{A}_{p, r}$. Let $K \geq 5$ be a sufficiently large constant and $\varepsilon \in(0,1)$ a sufficiently small constant, both depending only on the dimension $d$. Let $B=B(x, \theta)$ with $x \in \mathbb{S}^{d-1}$ and $\frac{K}{n}<\theta \leq \frac{\varepsilon}{K}$. Let $x_{2} \in \mathbb{S}^{d-1}$ be such that 
$4 \theta \leq \rho\left(x, x_{2}\right) \leq K \theta \leq \varepsilon$. Let $B_{2}:=B\left(x_{2}, \theta\right)$. Then for a nonnegative function $f$ supported in $B:=B(x, \theta)$, and an arbitrary $z \in B_{2}$, we have

$$
\begin{aligned}
\left|\left(-\Delta_{0}\right)^{r / 2} V_{n} f(z)\right| & =\left|\int_{B} f(y) K_{n, r}(z \cdot y) d \sigma(y)\right| \sim \int_{B} f(y) \rho(z, y)^{-(d-1+r)} d \sigma(y) \\
& \sim \theta^{-r} \frac{1}{|B|} \int_{B} f(y) d \sigma(y) \equiv \theta^{-r} f_{B},
\end{aligned}
$$

where we used Lemma9.3 in the second step. On the other hand, using the weighted Bernstein inequality (5.5), we obtain

$$
\begin{aligned}
\left|\theta^{-r} f_{B}\right|^{p} w_{n}\left(B_{2}\right) & \leq c\left\|\left(-\Delta_{0}\right)^{r / 2} V_{n} f\right\|_{p, w_{n}}^{p} \leq c n^{r p}\left\|V_{n} f\right\|_{p, w_{n}}^{p} \\
& \leq c n^{r p}\|f\|_{p, w_{n}}^{p} .
\end{aligned}
$$

Thus, for any nonnegative function $f$ supported in $B$,

$$
\left|f_{B}\right|^{p} w_{n}\left(B_{2}\right) \leq c(n \theta)^{r p} \int_{B}|f(y)|^{p} w_{n}(y) d \sigma(y) .
$$

Since $w$ is a doubling weight, $w_{n}$ satisfies the doubling condition as well with $L_{w_{n}} \leq$ $c L_{w}$. Since $B \subset B\left(x_{2}, 2 K \theta\right)=2 K B_{2}$, it follows that

$$
w_{n}\left(B_{2}\right) \geq c w_{n}\left(2 K B_{2}\right) \geq c w_{n}(B) .
$$

This combined with (6.5) yields

$$
\left|f_{B}\right|^{p} \leq c(n \theta)^{r p} \frac{1}{w_{n}(B)} \int_{B}|f(y)|^{p} w_{n}(y) d \sigma(y) .
$$

Letting $f(y)=w_{n}(y)^{-\frac{p^{\prime}}{p}} \chi_{B}(y)$, we conclude that

$$
\frac{w_{n}(B)}{|B|}\left(\frac{1}{|B|} \int_{B} w_{n}(y)^{-\frac{p^{\prime}}{p}} d y\right)^{p-1} \leq c(1+n \theta)^{r p}
$$

whenever $B=B(x, \theta)$ with $n^{-1} K \leq \theta \leq \varepsilon / K$. On the other hand, since $w_{n}(x) \sim$ $w_{n}(y)$ whenever $\rho(x, y) \leq c n^{-1}$, (6.6) holds trivially if $n \theta \leq K$.

Next, we show (6.6) for the case of $B=B(x, \theta)$ and $\varepsilon / K \leq \theta \leq \pi$. We first observe that

$$
\frac{w_{n}(B)}{|B|}\left(\frac{1}{|B|} \int_{B} w_{n}(y)^{-\frac{p^{\prime}}{p}} d \sigma(y)\right)^{p-1} \leq c_{\varepsilon}\left(\int_{B} w_{n}(y)^{-\frac{1}{p-1}} d \sigma(y)\right)^{p-1} .
$$

Since the ball $B=B(x, \theta)$ can be covered by a number of $\leq C_{d} \varepsilon^{-d+1}$ spherical caps of radius $\leq \varepsilon / K$, it follows that

$$
\int_{B} w_{n}(y)^{-\frac{1}{p-1}} d \sigma(y) \leq C_{\varepsilon} \sup _{\operatorname{rad}\left(B^{\prime}\right)=\varepsilon / K} \int_{B^{\prime}} w_{n}(y)^{-\frac{1}{p-1}} d \sigma(y) .
$$

On the other hand, using the doubling condition, it is easily seen that if $B^{\prime}$ is a spherical cap with radius $\varepsilon / K$, then $w_{n}\left(B^{\prime}\right) \geq c_{\varepsilon} w_{n}\left(\mathbb{S}^{d-1}\right) \geq c_{\varepsilon}^{\prime}>0$. Therefore we get

$$
\begin{aligned}
\left(\int_{B} w_{n}(y)^{-\frac{1}{p-1}} d \sigma(y)\right)^{p-1} & \leq C_{\varepsilon} \sup _{\operatorname{rad}\left(B^{\prime}\right)=\varepsilon / K}\left(\int_{B^{\prime}} w_{n}(y)^{-\frac{1}{p-1}} d \sigma(y)\right)^{p-1} \\
& \leq C_{\varepsilon} \sup _{\operatorname{rad}\left(B^{\prime}\right)=\varepsilon / K}\left(\frac{1}{\left|B^{\prime}\right|} \int_{B^{\prime}} w_{n}(y)^{-\frac{1}{p-1}} d \sigma(y)\right)^{p-1} \frac{w_{n}\left(B^{\prime}\right)}{\left|B^{\prime}\right|} \\
& \leq c n^{r p} \leq c(n \theta)^{r p},
\end{aligned}
$$


where the third step uses (6.6) for the already proven case $\theta=\varepsilon / K$. This completes the proof of necessity.

To show the sufficiency, we assume that $w \in \mathbb{A}_{p, \tau}$ and $r>\tau$. Then for $f \in \Pi_{n}^{d}$,

$$
\left(-\Delta_{0}\right)^{r / 2} f(x)=\left(-\Delta_{0}\right)^{r / 2} V_{n} f(x)=\int_{\mathbb{S}^{d-1}} f(y) K_{n, r}(x \cdot y) d \sigma(y) .
$$

Using Lemma 9.2 and integration by parts, we have

$$
\begin{aligned}
\left|\left(-\Delta_{0}\right)^{r / 2} f(x)\right| & \leq c n^{d-1+r} \int_{\mathbb{S}^{d-1}}|f(y)|(1+n \rho(x, y))^{-(d-1+r)} d \sigma(y) \\
& \leq c\|f\|_{1}+c n^{1+r} \int_{0}^{\pi}(1+n \theta)^{-(1+r)}\left|f_{B(x, \theta)}\right| d \theta, \\
& \leq c n^{r}\|f\|_{p, w_{n}}+c J(x),
\end{aligned}
$$

where $J(x):=n^{1+r} \int_{0}^{\pi}(1+n \theta)^{-(1+r)}\left|f_{B(x, \theta)}\right| d \theta$. To estimate $J(x)$, we let Let $r_{1} \in(\tau, r)$, and choose $\alpha, \beta$ so that $\alpha+\beta=1+r, \alpha>r_{1}+\frac{1}{p}$ and $\beta>\frac{1}{p^{\prime}}:=1-\frac{1}{p}$. Then using Hölder's inequality, we obtain

$$
\begin{aligned}
J(x)^{p} & \leq c n^{(1+r) p}\left(\int_{0}^{\pi}(1+n \theta)^{-\alpha p}\left|f_{B(x, \theta)}\right|^{p} d \theta\right)\left(\int_{0}^{\pi}(1+n \theta)^{-\beta p^{\prime}} d \sigma(y)\right)^{p-1} \\
& \leq c n^{r p+1} \int_{0}^{\pi}(1+n \theta)^{-\alpha p+r_{1} p} \frac{1}{w_{n}(B(x, \theta))} \int_{B(x, \theta)}|f(y)|^{p} w_{n}(y) d \sigma(y) d \theta,
\end{aligned}
$$

where we used Assertion (ii) of Lemma 6.2 in the second step. For $\theta \in(0, \pi)$, let $\Lambda_{\theta}$ be a maximal $\theta$-separated subset of $\mathbb{S}^{d-1}$. Then

$$
\begin{aligned}
& \int_{\mathbb{S}^{d-1}}\left[\frac{1}{w_{n}(B(x, \theta))} \int_{B(x, \theta)}|f(y)|^{p} w_{n}(y) d \sigma(y)\right] w_{n}(x) d \sigma(x) \\
& \leq \sum_{\omega \in \Lambda_{\theta}} \int_{B(\omega, \theta)}\left[\frac{1}{w_{n}(B(x, \theta))} \int_{B(x, \theta)}|f(y)|^{p} w_{n}(y) d \sigma(y)\right] w_{n}(x) d \sigma(x) \\
& \leq c \sum_{\omega \in \Lambda_{\theta}} \int_{B(\omega, \theta)}\left[\frac{1}{w_{n}(B(\omega, \theta))} \int_{B(\omega, 3 \theta)}|f(y)|^{p} w_{n}(y) d \sigma(y)\right] w_{n}(x) d \sigma(x) \\
& \leq c \sum_{\omega \in \Lambda_{\theta}} \int_{B(\omega, 3 \theta)}|f(y)|^{p} w_{n}(y) d \sigma(y) \leq c\|f\|_{p, w_{n}}^{p},
\end{aligned}
$$

where the third step uses the doubling condition of $w_{n}$. Thus,

$$
\|J\|_{p, w_{n}}^{p} \leq c n^{r p+1} \int_{0}^{\pi}(1+n \theta)^{-\alpha p+r_{1} p} d \theta\|f\|_{p, w_{n}}^{p} \leq c n^{r p}\|f\|_{p, w_{n}}^{p} .
$$

This completes the proof of the sufficiency.

Theorem 6.4 implies the following interesting corollary on the weighted Bernstein inequality with respect to doubling weights.

Corollary 6.6. Given a doubling weight $w$ on $\mathbb{S}^{d-1}$ with $d \geq 3$, if the weighted Bernstein inequality (5.5) holds for some $p=p_{1} \in[1, \infty)$ and some positive number $r=r_{1}$ which is not an even integer, then automatically, it holds for all $p_{1} \leq p<\infty$ and $r \geq r_{1}$.

Proof. Firstly, note that from the proof of Theorem 6.4 if (5.5) holds for $p=p_{1} \in$ $[1, \infty)$ and $r=r_{1} \notin 2 \mathbb{N}$, then $w \in \mathbb{A}_{p_{1}, r_{1}}$. Since $\mathbb{A}_{p_{1}, r_{1}} \subset \mathbb{A}_{p, r}$ for all $p \geq p_{1}$ and $r \geq r_{1}$, Theorem 6.4 implies that (5.5) holds for all $p_{1} \leq p<\infty$ and $r>r_{1}$. Thus, 
it remains to show (5.5) for the case of $r=r_{1}$ and $p_{1}<p<\infty$. To see this, we first note that for all $F \in L_{p_{1}}$,

$$
\left\|\left(-\Delta_{0}\right)^{r_{1} / 2} V_{n} F\right\|_{p_{1}, w} \leq C n^{r_{1}}\left\|V_{n} F\right\|_{p_{1}, w} \leq C n^{r_{1}}\left\|V_{n} F\right\|_{p, w_{n}} \leq C n^{r_{1}}\|F\|_{p, w_{n}},
$$

where we used (5.5) with $r=r_{1}$ and $p=p_{1}$ in the first step, Theorem 3.2 in the second step, and Lemma 6.3 in the last step. On the other hand, using the unweighted Bernstein inequality, and the boundedness of the operator $V_{n}$ on $L_{\infty}$,

$$
\left\|\left(-\Delta_{0}\right)^{r_{1} / 2} V_{n} F\right\|_{\infty} \leq C n^{r_{1}}\left\|V_{n} F\right\|_{\infty} \leq C n^{r_{1}}\|F\|_{\infty}, \quad \forall F \in L_{\infty} .
$$

Thus, applying the Riesz-Thorin interpolation theorem, we deduce that

$$
\left\|\left(-\Delta_{0}\right)^{r_{1} / 2} V_{n} F\right\|_{p, w} \leq C n^{r_{1}}\|F\|_{p, w_{n}}, \quad \forall F \in L_{p}, \quad p_{1} \leq p \leq \infty .
$$

To complete the proof, we just note that $V_{n} f=f$ and $\|f\|_{p, w} \sim\|f\|_{p, w_{n}}$ for all $f \in \Pi_{n}^{d}$.

We conclude this section with the following example.

Example 6.7. Let $w(x)=\prod_{j=1}^{d}\left|x_{j}\right|^{\alpha_{j}}$ and $1 \leq p<\infty$. From the proof of Proposition 6.1 in [10], it is easy to verify that if $\min _{1 \leq j \leq d} \alpha_{j}>p-1$, then $w \in \mathbb{A}_{p, \tau}$, but $w \notin \mathbb{A}_{p, \xi}$ for any $\xi<\tau_{w, p}$, where

$$
\tau_{w, p}:=\frac{s_{w}}{p}-(d-1)=\frac{1}{p}\left(\sum_{j=1}^{d} \alpha_{j}-\min _{1 \leq j \leq d} \alpha_{j}\right)-\left(1-\frac{1}{p}\right)(d-1) .
$$

Thus, in this case, the weighted Bernstein inequality (5.5) holds for $r>\tau_{w, p}$, and fails for $0<r<\tau_{w, p}$.

\section{Approximation in $L^{p}$-Spaces With $0<p<1$}

Recall that the generalized de la Vallée Poussin mean $V_{n} f$ is defined by (2.10) for all $f \in L_{p}$ with $1 \leq p \leq \infty$. It can be easily seen from the definition that $V_{n} g=g$ for $g \in \Pi_{n}^{d}$, and for all $f \in L_{p}$ with $1 \leq p \leq \infty$,

$$
E_{2 n}(f)_{p} \leq\left\|f-V_{n} f\right\|_{p} \leq C_{d} E_{n}(f)_{p} .
$$

This last fact, however, cannot be true for $0<p<1$, in which case, $V_{n} f$ is not even defined for all $f \in L_{p}$. In this section, we shall prove that given a function $f \in L_{p}$ with $0<p<1$, there always exists a Fourier-Laplace series on $\mathbb{S}^{d-1}$ whose generalized de la Vallée Poussin mean converges to $f$ in $L_{p}$-norm, and an estimate weaker than (7.1) remains true. The idea of using generalized de la Vallée Poussin means of Fourier series to approximate functions in $L_{p}$ with $0<p<1$ goes back to Oswald 23.

Given a Fourier-Laplace series

$$
\sigma \sim \sum_{k=0}^{\infty} Y_{k}(x), \quad Y_{k} \in \mathcal{H}_{k}^{d}
$$

we define $V_{n} \sigma:=\sum_{k=0}^{2 n} \eta\left(\frac{k}{n}\right) Y_{k}(x)$, and and $S_{n} \sigma:=\sum_{k=0}^{n} Y_{k}(x)$.

Our main result in this section is the following. 
Theorem 7.1. If $0<p<1$ and $f \in L^{p}\left(\mathbb{S}^{d-1}\right)$, then there exists a Fourier-Laplace series $\sigma$ of the form (7.2) such that

$$
\left\|f-V_{n} \sigma\right\|_{p} \leq C_{p} n^{-(d-1)\left(\frac{1}{p}-1\right)}\left(\sum_{k=1}^{n} k^{d-2-(d-1) p} E_{k}(f)_{p}^{p}\right)^{\frac{1}{p}} .
$$

If, in addition,

$$
\sum_{k=1}^{\infty} k^{d-2-(d-1) p} E_{k}(f)_{p}^{p}<\infty
$$

then $f \in L_{1}$, and one has the following stronger estimate:

$$
\left\|f-V_{n} f\right\|_{p} \leq C n^{-(d-1)\left(\frac{1}{p}-1\right)}\left(\sum_{k=n+1}^{\infty} k^{(d-2)-(d-1) p} E_{k}(f)_{p}^{p}\right)^{\frac{1}{p}} .
$$

Remark 7.2. It is worth mentioning that the term on the right-hand side of (7.3) tends to 0 as $n \rightarrow \infty$ and therefore (7.3) can be considered as a generalization of Oswald's result [23] on $\mathbb{S}^{d-1}$.

In the case of periodic functions, Theorem 7.1 is due to Belinskii and Liflyand [3].

The proof of Theorem [7.1 relies on several lemmas.

Lemma 7.3. Assume that $f \in \Pi_{6 n}^{d}$, and $G_{n}:[-1,1] \rightarrow \mathbb{R}$ is an algebraic polynomial of degree at most $n$. If $0<p<1$, then

$$
\left(\int_{\mathbb{S}^{d-1}}\left|\int_{\mathbb{S}^{d-1}} f(y) G_{n}(x \cdot y) d \sigma(y)\right|^{p} d \sigma(x)\right)^{\frac{1}{p}} \leq C n^{\left(\frac{1}{p}-1\right)(d-1)}\|f\|_{p}\left\|G_{n}\right\|_{p, \alpha, \beta},
$$

where $\alpha=\beta=\frac{d-3}{2}$.

Proof. The desired inequality (7.5) follows directly from Lemma 3.7 applied to $w=1, q=1$ and $0<p<1$ :

$$
\begin{aligned}
\int_{\mathbb{S}^{d-1}} \mid \int_{\mathbb{S}^{d-1}} f(y) G_{n}(x \cdot y) & \left.d \sigma(y)\right|^{p} d \sigma(x) \\
\leq & C n^{(d-1)(1-p)} \int_{\mathbb{S}^{d-1}} \int_{\mathbb{S}^{d-1}}\left|f(y) G_{n}(x \cdot y)\right|^{p} d \sigma(y) d \sigma(x) \\
& =C n^{(d-1)(1-p)}\|f\|_{p}^{p}\left\|G_{n}\right\|_{p, \alpha, \beta}^{p} .
\end{aligned}
$$

Lemma 7.4. If $0<p<1$, and $f \in \Pi_{6 n}^{d}$, then

$$
\left\|V_{n} f\right\|_{p} \leq C_{p}\|f\|_{p}
$$

Proof. By (2.10), we have

$$
V_{n} f(x)=\int_{\mathbb{S}^{d-1}} f(y) K_{n}(x \cdot y) d \sigma(y)
$$

where $K_{n}=G_{n}^{\left(\frac{d-3}{2}, \frac{d-3}{2}\right)}$ is given by (2.11). Thus, using Lemma 7.3 with $\alpha=\beta=$ $\frac{d-3}{2}$, we deduce

$$
\left\|V_{n} f\right\|_{p} \leq C n^{\left(\frac{1}{p}-1\right)(d-1)}\|f\|_{p}\left\|G_{n}^{(\alpha, \beta)}\right\|_{p, \alpha, \beta} \leq C\|f\|_{p},
$$

where the last step uses (9.7). 
The following lemma plays a crucial role in the proof of Theorem 7.1

Lemma 7.5. Assume that $f \in \Pi_{k}^{d}$, and $0<p<1$. Then there exists a FourierLaplace series of the form (7.2) such that $S_{k} \sigma=f$, and for all $n \geq k$,

$$
\left\|V_{n} \sigma\right\|_{p} \leq C\left(\frac{k}{n}\right)^{(d-1)\left(\frac{1}{p}-1\right)}\|f\|_{p}
$$

Proof. Let $\Lambda_{k}$ be a maximal $\frac{\delta}{k}$-separated subset of $\mathbb{S}^{d-1}$, with $\delta \in(0,1)$ being a small constant depending only on $d$. We denote by $N_{k}$ the number of points in the set $\Lambda_{k}$. Then $N_{k} \sim k^{d-1}$, and by Lemma 3.6, there exists a positive cubature formula of degree $k$ on $\mathbb{S}^{d-1}$,

$$
\int_{\mathbb{S}^{d-1}} P(y) d \sigma(y)=\sum_{\omega \in \Lambda_{k}} \lambda_{\omega} P(\omega), \quad \forall P \in \Pi_{k}^{d}
$$

such that $\lambda_{\omega} \sim N_{k}^{-1}$ for all $\omega \in \Lambda_{k}$. Define

$$
\sigma(x):=\sum_{j=0}^{\infty} \sum_{\omega \in \Lambda_{k}} \lambda_{\omega} E_{j}^{\left(\frac{d-3}{2}, \frac{d-3}{2}\right)}(x \cdot \omega) f(x) .
$$

Clearly, by the cubature formula (7.7),

$$
\begin{aligned}
S_{k} \sigma & =\sum_{j=0}^{k}\left[\sum_{\omega \in \Lambda_{k}} \lambda_{\omega} E_{j}^{\left(\frac{d-3}{2}, \frac{d-3}{2}\right)}(x \cdot \omega)\right] f(x) \\
& =f(x) \sum_{j=0}^{k} \int_{\mathbb{S}^{d-1}} E_{j}^{\left(\frac{d-3}{2}, \frac{d-3}{2}\right)}(x \cdot y) d \sigma(y)=f(x) .
\end{aligned}
$$

Since for each $\omega \in \Lambda_{k}, E_{j}^{\left(\frac{d-3}{2}, \frac{d-3}{2}\right)}(x \cdot \omega)$, as a function of $x \in \mathbb{S}^{d-1}$, is a spherical harmonic of degree $j$, it follows that

$$
E_{j}^{\left(\frac{d-3}{2}, \frac{d-3}{2}\right)}(x \cdot \omega) f(x) \in \sum_{\ell=|k-j|}^{k+j} \mathcal{H}_{\ell}^{d} .
$$

We can rewrite (7.8) in the form $\sum_{m=0}^{\infty} Y_{m}(x)$, with

$$
Y_{m}(x)=\sum_{j=0}^{m+k} \frac{1}{N_{k}} \sum_{\omega \in \Lambda_{k}} \operatorname{proj}_{m}\left[f E_{j}^{\left(\frac{d-3}{2}, \frac{d-3}{2}\right)}(\langle\cdot, \omega\rangle)\right](x) \in \mathcal{H}_{m}^{d} .
$$

This also implies that $V_{n}^{(x)}\left[E_{j}^{\left(\frac{d-3}{2}, \frac{d-3}{2}\right)}(x \cdot \omega) f(x)\right]=0$ whenever $j \geq 2 n+k$, where we use the notation $V_{n}^{(x)}$ to mean that the operator $V_{n}$ acts on the variable $x$. Therefore, setting $P_{j}=E_{j}^{\left(\frac{d-3}{2}, \frac{d-3}{2}\right)}$, we obtain

$$
\begin{aligned}
V_{n} \sigma & =\frac{1}{N_{k}} \sum_{\omega \in \Lambda_{k}} V_{n}^{(x)}\left[\sum_{j=0}^{2 n+k} P_{j}(x \cdot \omega) f(x)\right]=\frac{1}{N_{k}} \sum_{\omega \in \Lambda_{k}} V_{n}^{(x)}\left[\sum_{j=0}^{6 n} \eta\left(\frac{j}{3 n}\right) P_{j}(x \cdot \omega) f(x)\right] \\
& =\frac{1}{N_{k}} \sum_{\omega \in \Lambda_{k}} V_{n}^{(x)}\left[K_{3 n}(x \cdot \omega) f(x)\right]
\end{aligned}
$$


where the function $K_{3 n}$ is defined by (2.11). Letting $\xi>\frac{d-1}{p}$, we have

$$
\begin{aligned}
\left\|V_{n} \sigma\right\|_{p}^{p} & \leq C N_{k}^{-p} \sum_{\omega \in \Lambda_{k}} \int_{\mathbb{S}^{d-1}}\left|K_{3 n}(x \cdot \omega) f(x)\right|^{p} d \sigma(x) \\
& \leq C\left(N_{k}^{-p} \sum_{\omega \in \Lambda_{k}}\left|f_{k, \xi}^{*}(\omega)\right|^{p}\right) \sup _{y \in \mathbb{S}^{d-1}} \int_{\mathbb{S}^{d-1}}\left|K_{3 n}(x \cdot y)\right|^{p}(1+k \rho(x, y))^{\xi p} d \sigma(x) \\
& \leq C N_{k}^{1-p}\left\|f_{k, \xi}^{*}\right\|_{p}^{p} \sup _{y \in \mathbb{S}^{d-1}} \int_{\mathbb{S}^{d-1}}\left|K_{3 n}(x \cdot y)\right|^{p}(1+n \rho(x, y))^{\xi p} d \sigma(x) \\
& \leq C k^{(d-1)(1-p)} n^{(d-1)(p-1)}\|f\|_{p}^{p}=C\left(\frac{k}{n}\right)^{(d-1)(1-p)}\|f\|_{p}^{p},
\end{aligned}
$$

where we used Lemma 7.4 in the first step, the maximal function defined by (3.7) in the second step, and Theorem 3.4 and Lemma 2.1 in the last step. This completes the proof.

Lemma 7.6. If $0<p<1$ and $f \in L^{p}\left(\mathbb{S}^{d-1}\right)$ satisfies (7.4), then $f \in L\left(\mathbb{S}^{d-1}\right)$ and

$$
\int_{\mathbb{S}^{d-1}}|f(x)| d \sigma(x) \leq C\left(\sum_{k=1}^{\infty} k^{d-2-(d-1) p} E_{k}(f)_{p}^{p}\right)^{\frac{1}{p}}+C\|f\|_{p}
$$

Proof. Let $f_{j} \in \Pi_{2^{j}}^{d}$ be such that $E_{2^{j}}(f)_{p}:=\left\|f-f_{j}\right\|_{p}$ for $j \geq 0$. Then by Fatou's lemma, we have

$$
\begin{aligned}
\|f\|_{1} & \leq\left\|f_{0}\right\|_{1}+\sum_{j=1}^{\infty}\left\|f_{j}-f_{j-1}\right\|_{1} \leq C\|f\|_{p}+C \sum_{j=1}^{\infty} 2^{j(d-1)\left(\frac{1}{p}-1\right)}\left\|f_{j}-f_{j-1}\right\|_{p} \\
& \leq C\|f\|_{p}+C \sum_{j=1}^{\infty} 2^{j(d-1)\left(\frac{1}{p}-1\right)} E_{2^{j-1}}(f)_{p} \leq C\|f\|_{p}+C\left(\sum_{j=1}^{\infty} 2^{j(d-1)(1-p)} E_{2^{j-1}}(f)_{p}^{p}\right)^{\frac{1}{p}} \\
& \leq C\|f\|_{p}+C\left(\sum_{k=1}^{\infty} k^{(d-1)(1-p)-1} E_{k}(f)_{p}^{p}\right)^{\frac{1}{p}}<\infty
\end{aligned}
$$

where the second step uses the Nikolskii inequality.

We are now in a position to prove Theorem 7.1

Proof of Theorem 7.1. Assume that $2^{m-1} \leq n<2^{m}$. Let $f_{2^{j}} \in \Pi_{2^{j}}^{d}$ be such that $\left\|f-f_{2^{j}}\right\|_{p}=E_{2^{j}}(f)_{p}$. Set $g_{0}=g_{0}$, and $g_{j}=f_{2^{j}}-f_{2^{j-1}} \in \Pi_{2^{j}}^{d}$ for $j \geq 1$. For each $g_{j}$, let $\sigma_{j}:=\sum_{k=0}^{\infty} Y_{j, k}, \quad Y_{j, k} \in \mathcal{H}_{k}^{d}$ be the Fourier-Laplace series built from Lemma 7.5. Thus, by Lemma 7.5 .

$$
S_{2^{j}} \sigma_{j}=\sum_{k=0}^{2^{j}} Y_{j, k}=g_{j}
$$

and for any $n \geq 2^{j}$,

$$
\left\|V_{n} \sigma_{j}\right\|_{p} \leq C\left(\frac{2^{j}}{n}\right)^{(d-1)\left(\frac{1}{p}-1\right)}\left\|g_{j}\right\|_{p} \leq C\left(\frac{2^{j}}{n}\right)^{(d-1)\left(\frac{1}{p}-1\right)} E_{2^{j-1}}(f)_{p} .
$$

Now define

$$
\sigma:=\sum_{k=0}^{\infty} Y_{k}(x):=f_{0}(x)-\sum_{j=1}^{\infty} \sum_{k>2^{j}} Y_{j, k}(x),
$$


where

$$
Y_{k}(x):=-\sum_{0 \leq j \leq \log _{2} k} Y_{j, k}(x), \text { for } k>1 .
$$

Then

$$
\begin{aligned}
V_{n} \sigma & =f_{0}-\sum_{j=1}^{m} V_{n}\left(\sum_{2^{j}<k \leq 2 n} Y_{j, k}\right)=f_{0}-\sum_{j=1}^{m} V_{n}\left(\sum_{k=0}^{2 n} Y_{j, k}\right)+\sum_{j=1}^{m} V_{n}\left(\sum_{k=0}^{2^{j}} Y_{j, k}\right) \\
& =f_{0}-\sum_{j=1}^{m} V_{n}\left(\sigma_{j}\right)+\sum_{j=1}^{m} V_{n}\left(g_{j}\right)=f_{0}-\sum_{j=1}^{m} V_{n}\left(\sigma_{j}\right)+\sum_{j=1}^{m-1} g_{j}+V_{n}\left(g_{m}\right) \\
& =f_{m-1}+V_{n}\left(g_{m}\right)-\sum_{j=1}^{m} V_{n}\left(\sigma_{j}\right) .
\end{aligned}
$$

It follows that

$$
\begin{aligned}
\left\|f-V_{n} \sigma\right\|_{p}^{p} & \leq\left\|f-f_{m-1}\right\|_{p}^{p}+\left\|V_{n}\left(g_{m}\right)\right\|_{p}^{p}+\sum_{j=1}^{m}\left\|V_{n}\left(\sigma_{j}\right)\right\|_{p}^{p} \\
& \leq C E_{2^{m-1}}(f)_{p}^{p}+C \sum_{j=1}^{m} 2^{(j-m)(1-p)(d-1)}\left\|g_{j}\right\|_{p}^{p} \\
& \leq C 2^{-m(1-p)(d-1)} \sum_{j=1}^{m} 2^{j(1-p)(d-1)} E_{2^{j}}(f)_{p}^{p} .
\end{aligned}
$$

Thus,

$$
\left\|f-V_{n} \sigma\right\|_{p} \leq C n^{-(d-1)\left(\frac{1}{p}-1\right)}\left(\sum_{k=1}^{n} k^{d-2-(d-1) p} E_{k}(f)_{p}^{p}\right)^{\frac{1}{p}} .
$$

This completes the proof of the first part.

To show the second part, we first observe that by Lemma 7.6, if $f$ satisfies (7.4), then it must be in $L^{1}\left(\mathbb{S}^{d-1}\right)$, and hence $V_{n} f$ is defined. It follows that

$$
\begin{aligned}
& \left\|f-V_{n} f\right\|_{p}^{p} \leq\left\|f-f_{2^{m-1}}\right\|_{p}^{p}+\left\|V_{n}\left(f_{2^{m-1}}\right)-V_{n} f\right\|_{p}^{p} \\
& \leq E_{2^{m-1}}(f)_{p}^{p}+\sum_{j=m}^{\infty}\left\|V_{n}\left(f_{2^{j}}-f_{2^{j-1}}\right)\right\|_{p}^{p} \leq E_{2^{m-1}}(f)_{p}^{p} \\
& +C \sum_{j=m}^{\infty}\left(2^{j}+n\right)^{(d-1)(1-p)} \int_{\mathbb{S}^{d-1}} \int_{\mathbb{S}^{d-1}}\left|K_{n}(x \cdot y)\right|^{p}\left|f_{2^{j}}(y)-f_{2^{j-1}}(y)\right|^{p} d \sigma(y) d \sigma(x) \\
& \leq E_{2^{m-1}}(f)_{p}^{p}+C \sum_{j=m}^{\infty} n^{(d-1)(p-1)} 2^{j(d-1)(1-p)} E_{2^{j-1}}(f)_{p}^{p} \\
& \leq C n^{-(d-1)(1-p)} \sum_{k=[n / 2]}^{\infty} k^{(d-1)(1-p)-1} E_{k}(f)_{p}^{p} .
\end{aligned}
$$

To complete the proof, we just need to observe that

$$
f-V_{n} f=(f-P)-V_{n}(f-P), \quad \forall P \in \Pi_{n}^{d} .
$$

Let us present a similar result for the moduli of continuity on the sphere $\omega(f, t)_{p}$ introduced by Ditzian $([12,13)$. Let $S O(d)$ denote the group of all $d \times d$ orthogonal 
matrices. Given $t \in(0, \pi)$, we denote by $O_{t}$ the class of matrices $\rho \in S O(d)$ such that $(\rho x \cdot x) \geq \cos t$ for all $x \in \mathbb{S}^{d-1}$. The first order modulus of continuity is then defined by

$$
\omega(f, t)_{p}=\sup _{\rho \in O_{t}}\left\|\Delta_{\rho} f\right\|_{L^{p}\left(\mathbb{S}^{d-1}\right)}
$$

where $\Delta_{\rho} f=f(\rho x)-f(x)$.

Using Theorem 7.1, and the Jackson inequality $E_{n}(f)_{p} \leq C_{p} \omega\left(f, n^{-1}\right)_{p}$ for $0<$ $p<1$ proved in [9, Theorem 4.1], we deduce the following corollary:

Corollary 7.7. If $f \in L^{p}\left(\mathbb{S}^{d-1}\right)$ with $0<p<1$ then there exists a Fourier-Laplace series $\sigma$ on the sphere which is summable to $f$ by the generalized de la Vallée Poussin means with the rate

$$
\left\|f-V_{n} \sigma\right\|_{p} \leq C_{p, d} n^{-(d-1)\left(\frac{1}{p}-1\right)}\left(\int_{n^{-1}}^{\pi} t^{(d-1) p-d} \omega(f, t)_{p}^{p} d t\right)^{\frac{1}{p}} .
$$

\section{Sobolev-Type EMBEDDing With Weights}

In this section we study an embedding theorem for weighted Besov spaces. Let $E_{n}(f)_{p, w}$ be the best approximation of $f \in L_{p, w}$ by spherical polynomials of degree at most $n$ in the $L_{p, w}$-metric. Given $0<p \leq \infty, \nu>0$ and $0<\tau \leq \infty$, the weighted Besov space $B_{\tau}^{\nu}\left(L_{p, w}\right)$ is the collection of all functions $f \in L_{p, w}$ with finite quasi-norm

$$
\|f\|_{B_{\tau}^{\nu}\left(L_{p, w}\right)}=\|f\|_{p, w}+\left(\sum_{j=0}^{\infty} 2^{j \nu \tau} E_{2^{j}}(f)_{p, w}^{\tau}\right)^{1 / \tau}
$$

with the usual change when $\tau=\infty$.

The following Sobolev-type embedding result for the Besov space on $\mathbb{R}^{d}$ with the limiting smoothness parameter is well known: $B_{q}^{r}\left(L_{p}\left(\mathbb{R}^{d}\right)\right) \hookrightarrow L^{q}\left(\mathbb{R}^{d}\right), r=$ $d\left(\frac{1}{p}-\frac{1}{q}\right)>0$ (see, e.g., [24, (8.2)]).

For functions on $\mathbb{S}^{d-1}$, it was shown in [10, Th. 2.5] that if $0<p<q \leq \infty$ and $w$ is doubling, then for $\nu>s_{w}\left(\frac{1}{p}-\frac{1}{q}\right)$ one has $B_{q}^{\nu}\left(L_{p, w}\right) \subset L_{q, w}$. In the unweighted case this result was obtained in [17, Cor. 4]. Our next theorem extends the previous results for the limiting smoothness parameter.

Theorem 8.1. If $0<p<q<\infty$ and $w$ is doubling, then for $\nu:=s_{w}\left(\frac{1}{p}-\frac{1}{q}\right)$ we have $B_{q}^{\nu}\left(L_{p, w}\right) \subset L_{q, w}$ and

$$
\|f\|_{q, w} \leq C\|f\|_{B_{q}^{\nu}\left(L_{p, w}\right)}
$$

for all $f \in B_{q}^{\nu}\left(L_{p, w}\right)$. Furthermore, if $0<p<\infty$ and $\nu=\frac{s_{w}}{p}$, then each function $f \in B_{\infty}^{\nu}\left(L_{p, w}\right)$ can be identified with a continuous function on $\mathbb{S}^{d-1}$.

For the proof of (8.1), we need the following lemma, which follows directly from 14. Lemma 4.2], and Lemma 3.7.

Lemma 8.2. Assume that $0<p<q \leq \infty$ and $f \in L_{p, w}$. Let $\left\{f_{2^{n}}\right\}_{n=1}^{\infty}$ be a sequence of spherical polynomials such that $f_{2^{n}} \in \Pi_{2^{n}}^{d}$, and $\left\|f-f_{2^{n}}\right\|_{p, w} \leq$ $C_{1} E_{2^{n}}(f)_{p, w}$ for each $n \in \mathbb{N}$ and some positive constant $C_{1}$. Then for any $N \in \mathbb{N}$,

$$
\left\|\sum_{n=1}^{N}\left(f_{2^{n}}-f_{2^{n-1}}\right)\right\|_{q, w} \leq C_{p, q, w}\left(\sum_{n=1}^{N}\left(2^{n s_{w}\left(\frac{1}{p}-\frac{1}{q}\right)} E_{2^{n}}(f)_{p, w}\right)^{q_{1}}\right)^{\frac{1}{q_{1}}}
$$


where

$$
q_{1}:= \begin{cases}q, & \text { if } 0<q<\infty \\ 1, & \text { if } q=\infty\end{cases}
$$

We point out that Lemma 4.2 of 14 applies to a more general setting, where the Nikolskii type inequality is applicable.

Now we are in a position to show Theorem 8.1

Proof of Theorem 8.1. The proof runs along the same lines as that in 14, Th. 4.1], but is different from those in [17, Cor. 4] and [10, Th.2.5]. Let $f_{2^{j}} \in \Pi_{2^{j}}^{d}$ be such that $E_{2^{j}}(f)_{p, w}=\left\|f-f_{2^{j}}\right\|_{p, w}$ for $j \geq 0$. Using Lemma 8.2, we obtain

$$
\left\|\sum_{j=1}^{N}\left(f_{2^{j}}-f_{2^{j-1}}\right)\right\|_{q, w} \leq C\left(\sum_{j=1}^{N}\left(\left(2^{j s_{w}\left(\frac{1}{p}-\frac{1}{q}\right)} E_{2^{j}}(f)_{p, w}\right)^{q_{1}}\right)^{\frac{1}{q_{1}}}, \forall N \in \mathbb{N} .\right.
$$

Since

$$
f=f_{1}+\sum_{j=1}^{\infty}\left(f_{2^{j}}-f_{2^{j-1}}\right),
$$

with the series converging in $L_{p, w}$-metric, it follows by Fatou's lemma and equivalence of different metrics on the finite-dimensional linear space $\Pi_{2}^{d}$ that for $q<\infty$

$$
\begin{aligned}
\|f\|_{q, w} & \leq C_{q}\left\|f_{1}\right\|_{q, w}+C_{q} \liminf _{N \rightarrow \infty}\left\|\sum_{j=1}^{N}\left(f_{2^{j}}-f_{2^{j-1}}\right)\right\|_{q, w} \\
& \leq C\left\|f_{1}\right\|_{p, w}+C\left(\sum_{j=1}^{\infty}\left(2^{j \nu} E_{2^{j}}(f)_{p, w}\right)^{q}\right)^{\frac{1}{q}} \\
& \leq C\|f\|_{p, w}+C\left(\sum_{j=1}^{\infty}\left(2^{j \nu} E_{2^{j}}(f)_{p, w}\right)^{q}\right)^{\frac{1}{q}} \sim\|f\|_{B_{q}^{\nu}\left(L_{p, w}\right)},
\end{aligned}
$$

where $\nu=s_{w}\left(\frac{1}{p}-\frac{1}{q}\right)$. A similar argument works equally well for the case $q=\infty$.

Given a doubling weight $w$, using (3.5), it is easily seen that

$$
\min _{x \in \mathbb{S}^{d-1}} w\left(B\left(x, n^{-1}\right)\right) \geq c_{w} n^{-s_{w}}, \quad \forall n \in \mathbb{N},
$$

where $c_{w}>0$ is independent of $n$ and $x$. We shall show that the index $\nu:=s_{w}\left(\frac{1}{p}-\frac{1}{q}\right)$ in Theorem 8.1 is sharp under the following additional assumption on the doubling weight $w$ :

$$
\min _{x \in \mathbb{S}^{d-1}} w\left(B\left(x, n^{-1}\right)\right) \leq c_{w}^{\prime} n^{-s_{w}}, \quad n=1,2, \cdots .
$$

More precisely, we shall prove that under the condition of (8.2), given any $0<\nu^{\prime}<$ $\nu:=s_{w}\left(\frac{1}{p}-\frac{1}{q}\right)$, there exists a function $f$ which satisfies $f \in B_{\tau}^{\nu^{\prime}}\left(L_{p, w}\right)$ for all $\tau>0$, but $f \notin L_{q, w}$. Indeed, conditions (8.1) and (8.2) imply that there exists a sequence of points $y_{n} \in \mathbb{S}^{d-1}$ such that

$$
w\left(B\left(y_{n}, n^{-1}\right)\right) \sim n^{-s_{w}}, \quad n=1,2, \cdots .
$$


On the other hand, by Lemma 4.6 of [7, there exists a sequence of positive spherical polynomials $f_{n}$ such that $f_{n} \in \Pi_{n}^{d}$ and

$$
f_{n}(x) \sim\left(1+n \rho\left(x, y_{n}\right)\right)^{-\ell}, \quad \forall x \in \mathbb{S}^{d-1},
$$

where $\ell$ is any given positive number greater than $\frac{1}{p}\left(s_{w}+d\right)$. A straightforward calculation, using (8.3) and (3.6), then shows that

$$
\left\|f_{n}\right\|_{p_{1}, w} \sim\left\|f_{n}\right\|_{p_{1}, w_{n}} \sim n^{-s_{w} / p_{1}}, \quad \forall p_{1} \geq p .
$$

Let

$$
f=\sum_{n=1}^{\infty} 2^{n s_{w} / q} 2^{n \varepsilon} f_{2^{n}}
$$

where $\varepsilon$ is a positive constant satisfying $0<\varepsilon<\nu-\nu^{\prime}$. Then, with $\theta:=\min \{p, 1\}$, we have

$$
E_{2^{n}}(f)_{p, w} \leq\left(\sum_{k \geq n} 2^{n \theta s_{w} / q} 2^{n \varepsilon \theta}\left\|f_{2^{n}}\right\|_{p, w}^{\theta}\right)^{\frac{1}{\theta}} \leq C 2^{-n s_{w}\left(\frac{1}{p}-\frac{1}{q}\right)+n \varepsilon}=C 2^{-n \nu} 2^{n \varepsilon} .
$$

Thus, for any $\tau>0$,

$$
\|f\|_{B_{\tau}^{\nu^{\prime}\left(L_{p, w}\right)}} \leq C\left(\sum_{n=1}^{\infty} 2^{n \nu^{\prime} \tau} 2^{-n \tau \nu} 2^{n \tau \varepsilon}\right)^{\frac{1}{\tau}}<\infty .
$$

In particular, this implies that the series (8.4) converges in $L_{p, w}$-metric. Next, we show that $f \notin L_{q, w}$. To see this, we note that each term $f_{2^{n}}$ in the series on the right hand side of (8.4) is nonnegative, thus, by the monotone convergence theorem, $f \in L_{q, w}$ if any only if the series on the right hand side of (8.4) converges in $L_{q, w}$-metric, but this is impossible, since

$$
2^{n s_{w} / q} 2^{n \varepsilon}\left\|f_{2^{n}}\right\|_{q, w} \sim 2^{n \varepsilon} \rightarrow \infty \text { as } n \rightarrow \infty .
$$

This completes the proof.

We conclude this section with the following remark.

Remark 8.3. It is very easy to verify that all weights of the form (3.4) satisfy the condition (8.2). In general, one can show that if a doubling weight $w$ satisfies the condition

$$
\min _{x \in \mathbb{S}^{d-1}} w\left(B\left(x, n^{-1}\right)\right) \sim n^{-\xi}, \quad n=1,2, \cdots,
$$

for some $\xi>0$, then the Nikolski inequality (3.10) and Theorem 8.1 with $s_{w}=\xi$ hold, and in both cases, the index $\nu:=\xi\left(\frac{1}{p}-\frac{1}{q}\right)$ is sharp.

\section{Appendix: Proof of Theorem 2.2}

The main purpose in this section is to prove Theorem 2.2. The proof relies on the following two lemmas. Let us recall that $\alpha \geq \beta \geq-\frac{1}{2}$.

Lemma 9.1. If $k$ is a nonnegative integer, and $\theta \in[0, \pi]$, then

$$
\frac{1}{2 k+\alpha+\beta+2} E_{k}^{(\alpha+1, \beta)}(\cos \theta)=\sum_{j=0}^{k} E_{j}^{(\alpha, \beta)}(\cos \theta), \quad \theta \in[0, \pi]
$$


and

$$
\left|E_{k}^{(\alpha, \beta)}(\cos \theta)\right| \leq \begin{cases}C k^{2 \alpha+1}, & \text { if } 0 \leq \theta \leq k^{-1} \\ C k^{\alpha+\frac{1}{2}} \theta^{-\alpha-\frac{1}{2}}(\pi-\theta)^{-\beta-\frac{1}{2}}, & \text { if } k^{-1} \leq \theta \leq \pi-k^{-1} \\ C k^{\alpha+\beta+1}, & \text { if } \pi-k^{-1}<\theta \leq \pi\end{cases}
$$

If, in addition, $\theta \in\left[0,(2 k)^{-1}\right]$, then

$$
E_{k}^{(\alpha, \beta)}(\cos \theta) \geq \frac{1}{2} E_{k}^{(\alpha, \beta)}(1) \sim k^{2 \alpha+1} .
$$

Proof. Equation (9.1) follows directly by [28, p. 257, (9.4.3)] while inequality (9.2) is a simple consequence of (2.3) and [28, (7.32.5), (4.1.3)] and the following fact:

$$
\frac{\Gamma(x+a)}{\Gamma(x)}=x^{a}+O\left(x^{a-1}\right) \quad \text { as } x \rightarrow \infty, \quad a \in \mathbb{R} .
$$

Finally, (9.3) follows directly from Bernstein's inequality:

$$
\left|E_{n}^{(\alpha, \beta)}(\cos \theta)-E_{n}^{(\alpha, \beta)}(1)\right| \leq n \theta\left\|E_{n}^{(\alpha, \beta)}\right\|_{\infty}=n \theta E_{n}^{(\alpha, \beta)}(1) .
$$

Lemma 9.2. If $r>0$, and $\theta \in[0, \pi]$, then

$$
\left|G_{n, r}(\cos \theta)\right| \leq c n^{2 \alpha+2+r}(1+n \theta)^{-(2 \alpha+2+r)} .
$$

Proof. Assume that $2^{m-1} \leq n<2^{m}$, and set $\psi(x)=\eta(x / 2)-\eta(x)$. Since

$$
\eta\left(\frac{k}{n}\right)=\eta\left(\frac{k}{2^{m+1}}\right) \eta\left(\frac{k}{n}\right)=\sum_{j=0}^{m}\left(\eta\left(\frac{k}{2^{j+1}}\right)-\eta\left(\frac{k}{2^{j}}\right)\right) \eta\left(\frac{k}{n}\right)+\eta(k) \eta\left(\frac{k}{n}\right),
$$

it follows that

$$
G_{n, r}(x)=\sum_{j=0}^{m+2} F_{j}(x)
$$

where $F_{0}(x)=(2+\alpha+\beta)^{\frac{r}{2}} E_{1}^{(\alpha, \beta)}(x)$, and

$$
F_{j}(x)=\sum_{k=2^{j}}^{2^{j+2}} \eta\left(\frac{k}{n}\right) \psi\left(\frac{k}{2^{j}}\right)(k(k+\alpha+\beta+1))^{\frac{r}{2}} E_{k}^{(\alpha, \beta)}(x) .
$$

Using Lemma 2.1 with $N=2^{j}$ and $\varphi(x)=\eta\left(\frac{2^{j} x}{n}\right) \psi(x)\left(x\left(x+2^{-j}(\alpha+\beta+1)\right)\right)^{\frac{r}{2}}$, we obtain

$$
\left|F_{j}(\cos \theta)\right| \leq C 2^{j(r+2 \alpha+2)}\left(1+2^{j} \theta\right)^{-\ell}, \quad \forall \ell>0 .
$$

Thus, choosing $\ell>r+2 \alpha+2$, we obtain

$$
\begin{aligned}
\left|G_{n, r}(\cos \theta)\right| & \leq c \sum_{j=0}^{m} 2^{j(r+2 \alpha+2)}\left(1+2^{j} \theta\right)^{-\ell} \\
& \leq c \sum_{0 \leq j \leq \min \left\{m, \log _{2} \theta^{-1}\right\}} 2^{j(r+2 \alpha+2)}+\sum_{\min \left\{m, \log _{2} \theta^{-1}\right\}<j \leq m} \theta^{-\ell} 2^{j(r+2 \alpha+2-\ell)} \\
& \leq c n^{2 \alpha+2+r}(1+n \theta)^{-2 \alpha-2-r} .
\end{aligned}
$$


Now we are in a position to prove Theorem 2.2 .

Proof of Theorem 2.2. We first show that

$$
\left\|G_{n}\right\|_{p, \alpha, \beta} \sim n^{(2 \alpha+2)\left(1-\frac{1}{p}\right)} .
$$

Indeed, the upper bound of (9.7) follows directly from Lemma 2.1 with $i=0$ and $\ell>\frac{2 \alpha+2}{p}$. On the other hand, using (9.3), we deduce

$$
G_{n}(\cos \theta) \geq \frac{1}{2} G_{n}(1) \sim n^{2 \alpha+2}, \quad \theta \in\left[0,(2 n)^{-1}\right] .
$$

This, in particular, implies

$$
\left\|G_{n}\right\|_{p, \alpha, \beta} \geq c n^{2 \alpha+2}\left(\int_{0}^{(2 n)^{-1}} t^{2 \alpha+1} d t\right)^{\frac{1}{p}} \sim n^{(2 \alpha+2)\left(1-\frac{1}{p}\right)}
$$

which gives the desired lower estimate of (9.7). Thus, the proof of (2.15) is reduced to showing that

$$
\left\|G_{n, r}\right\|_{p, \alpha, \beta} \sim \begin{cases}n^{r-(2 \alpha+2)\left(\frac{1}{p}-1\right)}, & \text { if } r>(2 \alpha+2)\left(\frac{1}{p}-1\right), \\ 1, & \text { if } r<(2 \alpha+2)\left(\frac{1}{p}-1\right), \text { and } r \notin \mathbb{N}, \\ \log ^{\frac{1}{p}} n, & \text { if } r=(2 \alpha+2)\left(\frac{1}{p}-1\right) \text { and } r \notin \mathbb{N} .\end{cases}
$$

The upper estimates of (9.8) follows directly from Lemma 9.2, while the proof of the desired lower estimates for the case of $r>(2 \alpha+2)\left(\frac{1}{p}-1\right)$ can be done almost identically as that of (9.7).

The lower estimates of (9.8) for the remaining cases can be deduced directly from the following crucial lemma, which is of independent interest.

Lemma 9.3. Let $r>0$, and assume that $r$ is not an even integer if $\alpha+\beta+1>0$, and $r$ is not an integer if $\alpha+\beta+1=0$. Then for any $\theta \in\left[A n^{-1}, \varepsilon\right]$,

$$
\left|G_{n, r}(\cos \theta)\right| \sim \theta^{-(2 \alpha+2+r)}, \quad \forall n \geq A \varepsilon^{-1},
$$

where $A$ and $\varepsilon$ denote a sufficiently large and, respectively, small positive constants, both depending only on $\alpha$ and $r$.

For the proof of Lemma 9.3, we need some well-known results for the Cesàro kernels of the Jacobi polynomial expansions, defined as follows:

$$
S_{n}^{\delta,(\alpha, \beta)}(x)=\frac{1}{A_{n}^{\delta}} \sum_{k=0}^{n} A_{n-k}^{\delta} E_{k}^{(\alpha, \beta)}(x), \quad \delta>0, \quad x \in[-1,1] .
$$

Lemma 9.4. (i) If $\delta \geq \alpha+\frac{3}{2}$ and $\theta \in\left[0, \frac{\pi}{2}\right]$, then

$$
\left|S_{n}^{\delta,(\alpha, \beta)}(\cos \theta)\right| \leq C n^{2 \alpha+2}(1+n \theta)^{-2 \alpha-3} .
$$

(ii) If $\delta \geq \alpha+\beta+2$, then the Cesàro $(C, \delta)$-kernels $S_{n}^{\delta,(\alpha, \beta)}$ are positive on $[-1,1]$; that is,

$$
S_{n}^{\delta,(\alpha, \beta)}(x) \geq 0, \quad x \in[-1,1] .
$$

The results of Lemma 9.4 are well known. Indeed, (9.10) can be found in 4 , Theorem 2.1], whereas (9.11) was proved in [2] and [16, (4.13)].

In summary, we have reduced the proof of (9.8) to showing Lemma 9.3 The proof of this lemma is given as follows: 
Proof of Lemma 9.3. The upper estimate of (9.9) has already been given in Lemma 9.2 So we only need to show the lower estimate of (9.9).

For simplicity, we assume that $\alpha+\beta+1>0$. The proof below with a slight modification works equally well for the case when $\alpha+\beta+1=0$ and $r$ is not an integer. Let $\ell$ be the smallest positive integer bigger than $\alpha+\beta+r+2$. Define $\ell+1$ functions $a_{n, r, j}:[0, \infty) \rightarrow \mathbb{R}, j=0,1, \cdots, \ell$ iteratively by

$$
\begin{aligned}
a_{n, r, 0}(s) & =(2 s+\alpha+\beta+1)(s(s+\alpha+\beta+1))^{\frac{r}{2}} \eta\left(\frac{s}{n}\right), \\
a_{n, r, j+1}(s) & =\frac{a_{n, r, j}(s)}{2 s+\alpha+\beta+j+1}-\frac{a_{n, r, j}(s+1)}{2 s+\alpha+\beta+j+3} \\
& =-\int_{0}^{1} \frac{d}{d t}\left[\frac{a_{n, r, j}(s+t)}{2(t+s)+\alpha+\beta+j+1}\right] d t, \quad j=0, \cdots, \ell-1 .
\end{aligned}
$$

Since $\eta$ equals 1 on $[0,1]$ and $\alpha+\beta+1>0$, using induction on $j$, it is easily seen that for $0 \leq j \leq \ell$,

$$
a_{n, r, j}(s)=\gamma_{r, j} s^{r+1-2 j}+s^{r-2 j} g_{j}\left(s^{-1}\right), \quad 1 \leq s \leq n-j,
$$

some functions $g_{j} \in C^{\infty}[0, \infty)$, where $\gamma_{r, 0}=2$, and $\gamma_{r, j}:=2^{1-j}(-1)^{j} r(r-2) \cdots(r-$ $2 j+2$ ) for $j \geq 1$. Moreover, a similar argument shows that

$$
\left|a_{n, r, j}(s)\right| \leq c_{j}(s+1)^{r+1-2 j}, \quad \forall s \geq 0 .
$$

Note that the constant $\gamma_{r, j}$ will never be zero if $r$ is not an even integer.

Next, using (9.1) and summation by parts $\ell$ times, we obtain

$$
G_{n, r}(t)=c \sum_{k=0}^{2 n} \frac{a_{n, r, \ell}(k)}{2 k+\alpha+\beta+\ell+1} E_{k}^{(\alpha+\ell, \beta)}(t),
$$

for some nonzero constant $c$ depending only on $\alpha$ and $\beta$. Let $v$ be the smallest positive integer greater than $\alpha+\beta+2$. Using summation by parts $v+1$ times, we deduce from (9.13) that

$$
G_{n, r}(t)=C \sum_{k=0}^{2 n}\left[\vec{\triangle}^{v+1} \frac{a_{n, r, \ell}(k)}{2 k+\alpha+\beta+\ell+1}\right] A_{k}^{v} S_{k}^{v,(\alpha+\ell, \beta)}(t),
$$

where $\vec{\triangle} \mu_{k}=\mu_{k}-\mu_{k+1}$ and $\vec{\triangle}^{i+1}=\vec{\triangle} \vec{\triangle}^{i}$. Setting

$$
\varphi(s)=\frac{a_{n, r, \ell}(s)}{2 s+\alpha+\beta+\ell+1},
$$

and using (9.12), we have, for $1 \leq s \leq n-\ell-v-1$,

$$
\varphi^{(v+1)}(s)=c_{v, \ell} s^{r-2 \ell-v-1}+s^{r-2 \ell-v-2} g\left(s^{-1}\right),
$$

where $g$ is a $C^{\infty}$-function on $[0, \infty)$, and

$$
c_{v, \ell}=2^{-1} \gamma_{r, \ell}(-1)^{v+1}(r-2 \ell+1)(r-2 \ell) \cdots(r-2 \ell-v) .
$$

It then follows that for $1 \leq k \leq n-2 \ell$,

$$
\begin{aligned}
\vec{\triangle}^{v+1} \varphi(k) & =(-1)^{v+1} \int_{[0,1]^{v+1}} \varphi^{(v+1)}\left(k+t_{1}+\cdots+t_{v+1}\right) d t_{1} \cdots d t_{v+1} \\
& =(-1)^{v+1} c_{v, \ell} k^{r-2 \ell-v-1}+O\left(k^{r-2 \ell-v-2}\right) .
\end{aligned}
$$


Since $r$ is not an even integer, and $2 \ell>r+1$, the constant $c_{v, \ell}$ is not zero. For $n-2 \ell \leq k \leq 2 n$, we have the following easy estimate

$$
\left|\vec{\triangle}^{v+1} \varphi(k)\right| \leq c k^{r-2 \ell-v-1}
$$

Thus, using (9.17) and (9.16), we may rewrite (9.14) in the form

$$
G_{n, r}(\cos \theta)=c \sum_{k=1}^{n-2 \ell} k^{r-2 \ell-v-1} A_{k}^{v} S_{k}^{v,(\alpha+\ell, \beta)}(\cos \theta)+R_{n, 1}(\theta)+R_{n, 2}(\theta),
$$

where $c \neq 0$, and

$$
\begin{aligned}
& \left|R_{n, 1}(\theta)\right| \leq C \sum_{k=[n / 2]}^{2 n} k^{r-2 \ell-1}\left|S_{k}^{v,(\alpha+\ell, \beta)}(\cos \theta)\right|, \\
& \left|R_{n, 2}(\theta)\right| \leq C \sum_{k=1}^{n} k^{r-2 \ell-2}\left|S_{k}^{v,(\alpha+\ell, \beta)}(\cos \theta)\right| .
\end{aligned}
$$

Since the Cesàro kernels $S_{k}^{v,(\alpha+\ell, \beta)}(\cos \theta)$ are positive by (9.11), it follows that for $\theta \in\left[A n^{-1}, \varepsilon\right]$,

$$
\begin{aligned}
& \sum_{k=1}^{n-2 \ell} k^{r-2 \ell-v-1} A_{k}^{v} S_{k}^{v,(\alpha+\ell, \beta)}(\cos \theta) \geq \sum_{1 \leq k \leq 2^{-1} \theta^{-1}} k^{r-2 \ell-v-1} A_{k}^{v} S_{k}^{v,(\alpha+\ell, \beta)}(\cos \theta) \\
& \quad \geq c \sum_{1 \leq k \leq 2^{-1} \theta^{-1}} k^{r+2 \alpha+1} \geq c_{1} \theta^{-(r+2 \alpha+2)}
\end{aligned}
$$

where we have used the positivity of $S_{k}^{v,(\alpha+\ell, \beta)}$ in the first step, and (9.3) in the second step.

To estimate the reminder term $R_{n, 1}(\theta)$, we use (9.10) and obtain

$$
\begin{aligned}
\left|R_{n, 1}(\theta)\right| & \leq C \sum_{k=[n / 2]}^{2 n} k^{r-2 \ell-1} k^{-1} \theta^{-(2 \alpha+2 \ell+3)} \leq n^{r-2 \ell-1} \theta^{-(2 \alpha+2 \ell+3)} \\
& =c(n \theta)^{r-2 \ell-1} \theta^{-(2 \alpha-r+2)} \leq c_{2} A^{-(2 \ell+1-r)} \theta^{-(2 \alpha-r+2)}
\end{aligned}
$$

provided that $n \theta \geq A$. Similarly, using (9.10), we have

$$
\begin{aligned}
\left|R_{n, 2}(\theta)\right| & \leq c \sum_{1 \leq k \leq \theta^{-1}} k^{r-2 \ell-2} k^{2(\alpha+\ell)+2}+c \sum_{\theta^{-1} \leq k \leq 2 n} k^{r-2 \ell-2} k^{-1} \theta^{-(2 \alpha+2 \ell+3)} \\
& \leq c \theta^{-(r+2 \alpha+1)}+c \theta^{-(r-2 \ell-2)} \theta^{-(2 \alpha+2 \ell+3)} \leq c \theta^{-(r+2 \alpha+1)} \leq c_{3} \varepsilon \theta^{-(r+2 \alpha+2)}
\end{aligned}
$$

provided that $\theta \leq \varepsilon$.

Putting these together, we conclude that for $\theta \in\left[n^{-1} A, \varepsilon\right]$,

$$
\left|G_{n, r}(\cos \theta)\right| \geq\left[c_{1}-c_{2} A^{-(2 \ell+1-r)}-c_{3} \varepsilon\right] \theta^{-(r+2 \alpha+2)} \geq c \theta^{-(r+2 \alpha+2)},
$$

provided that $A$ is large enough, and $\varepsilon$ is sufficiently small. This completes the proof. 


\section{REFERENCES}

[1] V. V. Arestov, On integral inequalities for trigonometric polynomials and their derivatives, Math. USSR, Izv. 18 (1982), 1-17; translation from Izv. Akad. Nauk SSSR, Ser. Mat. 45 (1981), 3-22.

[2] R. Askey and G. Gasper, Positive Jacobi polynomial sums II. Amer. J. Math. 98 (1976), no. 3, 709-737.

[3] E. Belinskii and E. Liflyand, Approximation properties in $L_{p}, 0<p<1$, Funct. Approx. Comment. Math. 22 (1993), 189-199.

[4] A. Bonami and J. L. Clerc, Sommes de Cesàro et multiplicateurs des dèveloppments en harmonique sphériques, Trans. Amer. Math. Soc. 183 (1973), 223-263.

[5] G. Brown and F. Dai, Approximation of smooth functions on compact two-point homogeneous spaces, J. Funct. Anal. 220 (2005), no. 2, 401-423.

[6] F. Dai, Strong convergence of spherical harmonic expansions on $H^{1}\left(S^{d-1}\right)$, Constr. Approx. 22 (2005), no. 3, 417-436.

[7] F. Dai, Multivariate polynomial inequalities with respect to doubling weights and $A_{\infty}$ weights, J. Funct. Anal. 235 (2006), no. 1, 137-170.

[8] F. Dai, Jackson-type inequality for doubling weights on the sphere, Constr. Approx. 24 (2006), 91-112.

[9] F. Dai and Z. Ditzian, Jackson theorem in $L_{p}, 0<p<1$, for functions on the sphere, Journal of Approx. Theory, 162 (2010), 382-391.

[10] F. Dai and H. P. Wang, Optimal cubature formulas in weighted Besov spaces with $A_{\infty}$ weights on multivariate domains, Constr. Approx., 37 (2013), 167-194.

[11] Z. Ditzian, Fractional derivatives and best approximation, Acta Math. Hungar., 81 (1998), 323-348.

[12] Z. Ditzian, A modulus of smoothness on the unit sphere, J. Anal. Math., 79 (1999), 189-200.

[13] Z. Ditzian, Jackson-type inequality on the sphere, Acta Math. Hungar., 102 (2004), (12), $1-35$.

[14] Z. Ditzian and S. Tikhonov, Ul'yanov and Nikol'skii-type inequalities, J. Approx. Theory, 133 (2005), 1, 100-133.

[15] T. Erdélyi, Notes on inequalities with doubling weights, J. Approx. Theory, 100 (1999), no. $1,60-72$.

[16] G. Gasper, Positive sums of the classical orthogonal polynomials, SIAM J. Math. Anal. 8 (1977), no. 3, 423-447.

[17] K. Hesse, H. N. Mhaskar, and I. H. Sloan, Quadrature in Besov spaces on the Euclidean sphere, J. of Complexity, 23 (2007), no. 4-6, 528-552.

[18] K. Ivanov, P. Petrushev, and Y. Xu, Sub-exponentially localized kernels and frames induced by orthogonal expansions, Math. Z., 264 (2010), 361-397.

[19] A. I. Kamzolov, Bernstein's inequality for fractional derivatives of polynomials in spherical harmonics, Russian Mathematical Surveys, 39(2) 1984, 163; translation from Uspekhi Mat. Nauk, 39 (1984), no. 2 (236), 159-160.

[20] A. K. Lerner, C. Pérez, A new characterization of the Muckenhoupt $A_{p}$ weights through an extension of the Lorentz-Shimogaki theorem, Indiana Univ. Math. J., 56 (2007), no. $6,2697-2722$.

[21] P. I. Lizorkin, Estimates for trigonometric and the Bernstein inequality for fractional derivatives, Izv. AN, Ser. Mat. 29 (1965) 109-126 (in Russian); translated in: Am. Math. Soc., Transl., 77 (1968), 45-62.

[22] G. Mastroianni and V. Totik, Weighted polynomial inequalities with doubling and $A_{\infty}$ weights, Constr. Approx. 16 (2000), no. 1, 37-71.

[23] P. Oswald, Rate of approximation by de la Vallee-Poussin means of trigonometric series in the metric of $L_{p}(0<p<1)$, Sov. J. Contemp. Math. Anal., Arm. Acad. Sci. 18 (1983), no. 3, 63-78; translation from Izv. Akad. Nauk Arm. SSR, Mat. 18 (1983), no. 3, 230-245.

[24] J. Peetre, Espaces dinterpolation et théeorème de Soboleff, Ann. Inst. Fourier (Grenoble), 16 (1966), 279-317.

[25] K. Runovskii and H.-J. Schmeisser, On some extensions of Bernstein's inequalities for trigonometric polynomials, Funct. et Approx. 29 (2004), 125-142. 
[26] K. Runovskii and H.-J. Schmeisser, Inequalities of Caldern-Zygmund type for trigonometric polynomials, Georgian Math. J., 8 (2001), no. 1, 165-179.

[27] E. M. Stein, Harmonic Analysis: Real-Variable Methods, Orthogonality, and Oscillatroy Integrals, Princeton Univ. Press, Princeton, NJ, 1993.

[28] G. Szegö, Orthogonal Polynomials, Amer. Math. Soc., New York, 1967.

[29] W. Trebels, Multipliers for $(C, \alpha)$-bounded Fourier expansions in Banach spaces and approximation theory, Lecture Notes in Mathematics, Springer, Vol. 329, 1973.

[30] K. Y. Wang and L. Q. Li, Harmonic Analysis and Approximation on the unit Sphere, Science Press, Beijing, 2000.

[31] J. Wu, Lower bounds for an integral involving fractional Laplacians and the generalized Navier-Stokes equations in Besov spaces, Commun. Math. Phys., 263 (2006), no. 3, 803831.

[32] J. Wu, Existence and uniqueness results for the 2-D dissipative quasi-geostrophic equation, Nonlinear Anal., Theory Methods Appl. 67 (2007), no. 11, 3013-3036.

Department of Mathematical and Statistical Sciences, University of Alberta, Edmonton, Alberta T6G 2G1, Canada.

E-mail address: fdai@ualberta.ca

iCRea and Centre de Recerca Matemàtica, Campus de Bellaterra, Edifici C 08193 Bellaterra (Barcelona), Spain.

E-mail address: stikhonov@crm.cat 